\title{
Cell face velocity alternatives in a structured colocated grid for the unsteady Navier-Stokes equations *
}

\author{
Antonio Pascau \\ Fluid Mechanics Group and LITEC, CSIC-University of Zaragoza, María de Luna \\ 3, 50018 Zaragoza, Spain
}

\begin{abstract}
The use of a colocated variable arrangement for the numerical solution of fluid flow is becoming more and more popular due to its coding simplicity. The inherent decoupling of the pressure and velocity fields in this arrangement can be handled via a special interpolation procedure for the calculation of the cell face velocity named PWIM (Pressure Weighted Interpolation Method). In this paper a discussion on the alternatives to extend PWIM to unsteady flows is presented along with a very simple criterium to ascertain if a given interpolation practice will produce steady results that are relaxation dependent or time step dependent. Following this criterium it will be shown that some prior schemes presented as time step independent are actually not, although by using special interpolations can be readily adapted to be. A systematic way of deriving different cell face velocity expressions will be presented and new formulae free of $\Delta t$ dependence will be derived. Several computational exercices will accompany the theoretical discussion to support our claims.
\end{abstract}

Key words: CFD, Colocated grid, Unsteady flow $P A C S: 47.11 .+\mathrm{j}$

* This work has been supported by Projects CIT-370200-2005-6 and ENE-200509214-204-03/ALT of The Spanish Government. It was carried out under the University of Zaragoza IRDI agreement with the Regional Government of Aragón to promote research activities.

Email address: pascau@unizar.es (Antonio Pascau). 


\section{Introduction}

It is well known that the arrangement of variables in a structured staggered grid produces a natural coupling between the velocity and pressure fields when a segregated approach is utilized. The $1 \Delta$ discretization of the pressure gradient in the velocity equation, and viceversa, the discretization of the velocity divergence in the pressure (or pressure correction) equation is very effective damping the fluctuations that may arise in the iterative process because one variable responds naturally to the perturbations of the other. However, the coupling is not perfect and many times some sort of underrelaxation is required to prevent overflow. On the other hand, the colocated arrangement causes a decoupling between both fields, as a $2 \Delta$ discretization arises in the continuity equation if no special interpolation is employed to calculate cell face velocities. In this sense the implementation of a staggered grid is more attractive as no additional care has to be taken to preserve a good PV coupling apart from underrelaxation. The ease of maintaining a stable velocity-pressure coupled iterative process is at the cost of making the coding more laborious since a different grid is required for each velocity component and for the pressure. If, to increase the convergence rate, a multigrid procedure is adopted, the whole implementation is prone to errors as the intergrid transfer operators are numerous and one has to write as many multigrid procedures as grids. Mainly due to this laborious multigrid coding there has been a growing tendency in the last two decades to use the colocated arrangement in which there is no multiplicity of prolongation or restriction interpolators.

Rhie and Chow showed how to deal with the PV coupling in a colocated arrangement in a seminal paper [1]. They put forward a special interpolation named PWIM (Pressure Weighted Interpolation Method) that maintains the PV coupling by calculating convecting cell face velocities as the weighted mean of adjacent nodal values plus an extra term which is a function of the pressure field. In the first applications of the procedure in the Navier-Stokes equations, which require underrelaxation, it was observed that the final solution was dependent on the underrelaxation factor. Majumdar [2] and Miller and Schmidt [3] showed that the correct implementation involved the storage of the face velocity from iteration to iteration in the same manner as the nodal velocity is stored. To make the solution independent of the underrelaxation factor, as it should be, a new term arises that contains the difference between the convective flux and the weighted nodal mean, both at the previous iteration. One of the problems encountered with the PWIM approach is that the pressure field can produce cell face values that if plotted with the resolved nodal values make the whole velocity field (nodes plus faces) look wiggly. This usually happens in regions where pressure gradient variations are significant. An interesting alternative based on a local solution was presented by Thiart [4] and slightly modified by Wang et. al. [5] that reduces the oscillations at 
the cost of increasing notably the computational burden. Later, a rather close interpolation to PWIM named MWIM was proposed by Aksoy and Chen [6]. The only difference between both approaches lies in the quantity chosen to be interpolated, a complete term interpolation, PWIM, or a factor-in-term interpolation, MWIM. This minor difference produces very similar results with both approaches, both suffering from the same oscillation problems in the fields. A very thorough review of all work published with both techniques up to the end of 1997 is provided by Miettinen [7], although he considers MWIM to differ from PWIM in a manner that is not consistent with MWIM original paper.

The main problem of the colocated arrangement, in connection with what was mentioned in the previous paragraph, is that there are two different velocity fields: the convecting velocity at the cell face and the convected velocity at the cell center. The former does not possess a discretized equation of its own but instead is calculated as a function of the nodal values of velocity and pressure. If the procedure is correctly implemented the convecting velocity satisfies continuity within machine accuracy unlike the convected velocity that due to the pressure field does not. In the PWIM approach the continuity error for the nodal velocities is approximately proportional to the fourth derivative of the pressure field and to the cell size to the third power ${ }^{1}$. So, in regions of large pressure variations a fine grid may be required to keep the mass imbalance for the cell center velocities below a certain tolerance.

The paper is mainly devoted to the discussion of cell face velocity alternatives for the (pseudo)unsteady Navier-Stokes equations solved following a segregated procedure with SIMPLE-family schemes. Its structure is as follows. We first describe the well known Rhie \& Chow interpolation in a steady case and its alternatives free of relaxation dependence. The application of SIMPLE, SIMPLEC and SIMPLER in a colocated grid will be described next because there has to be some discussion on interpolation issues and that affects both the pressure correction and the pressure equations. Then, the necessary changes for unsteady flow will be presented and several alternatives will be assessed, both new and old. Two sections are devoted to put forward the criterium to ascertain if a given scheme is time step dependent or not and to show how a non-consistent scheme can be converted into one free of $\Delta \mathrm{t}$ dependence. Finally some computational experiments will be shown to support our findings.

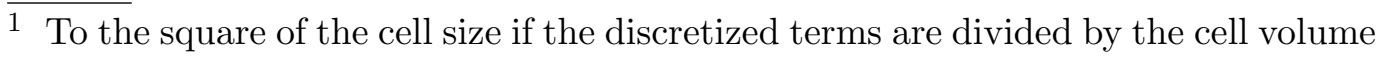




\section{Cell face velocity expression. Steady case}

In order to introduce the new procedure the detailed development of the east face velocity of a generic control volume associated to node $P$ will be explained. This is not the original way of presenting the PWIM approach, as Rhie-Chow way was rather heuristic, but the final expression is identical and this manner will allow us to derive clear conditions for freeing the solutions from relaxation dependency. There is a connection between consistent steady formulations and unsteady ones (the core of this paper) so we will start with a detailed description of the rationale behind the cell face velocity expression for a steady flow. Let $P$ and $E$ be the nodes that share the face $e$, all three along the $\mathrm{x}$ coordinate, and be $u$ the velocity component along the same direction. The convective flux is $\rho_{e} u_{e} A_{e}$ where $A_{e}$ depends on the geometry and dimensionality of the problem. The discretized momentum equation for $u_{P}$ is

$$
A_{P \mid P}^{u} u_{P}^{*}=\sum_{j \mid P} A_{j}^{u} u_{j}^{*}+S_{P}^{u} \Delta V_{P}-\left.\Delta V_{P} \frac{\partial p}{\partial x}\right|_{P}
$$

Subindices contain two letters when neccesary: the first one refers to the category/localization of the factor and the second one refers to the equation node. For instance, $A_{P \mid E}^{u}$ would mean the diagonal coefficient of the $u_{E}$ equation, and $\sum_{j \mid E}$ would represent the sum over all neighbours of the node E. The letter $j$ sweeps over all letters representing the neighbours of $E$ (for instance, $E E$ and $P$ in $1 \mathrm{D}) . S_{P}^{u}$ is the source per unit volume of the $P$ node. The terms in the discretized equation are the result of integrating over the control volume the convective, diffusive and source terms in the original differential equation. Let us note that we do not divide by the volume itself. The equation for the node $E$ is

$$
A_{P \mid E}^{u} u_{E}^{*}=\sum_{j \mid E} A_{j}^{u} u_{j}^{*}+S_{E}^{u} \Delta V_{E}-\left.\Delta V_{E} \frac{\partial p}{\partial x}\right|_{E}
$$

A similar fictitious equation can be written for the velocity at $e$

$$
A_{P \mid e}^{u} u_{e}^{*}=\sum_{j \mid e} A_{j}^{u} u_{j}^{*}+S_{e}^{u} \Delta V_{e}-\left.\Delta V_{e} \frac{\partial p}{\partial x}\right|_{e}
$$

It is worth noting that although this equation is neither assembled nor solved it should have the same formal structure as the nodal equations. The velocity $u_{e}$ is

$$
u_{e}^{*}=\frac{\sum_{j \mid e} A_{j}^{u} u_{j}^{*}+S_{e}^{u} \Delta V_{e}}{A_{P \mid e}^{u}}-\left.\frac{\Delta V_{e}}{A_{P \mid e}^{u}} \frac{\partial p}{\partial x}\right|_{e}=H_{e}^{u}-\left.\frac{\Delta V_{e}}{A_{P \mid e}^{u}} \frac{\partial p}{\partial x}\right|_{e}
$$


Rhie and Chow approach [1] can be interpreted as an interpolation that considers $H^{u}$ and $1 / A^{u}$ to be piecewise linear functions between nodes and hence the PWIM interpolation calculates

$$
\begin{aligned}
H_{e}^{u} & =\left(1-f_{x}\right) H_{E}^{u}+f_{x} H_{P}^{u}= \\
& =f_{x} \frac{\sum_{j \mid P} A_{j}^{u} u_{j}+S_{P}^{u} \Delta V_{P}}{A_{P \mid P}^{u}}+\left(1-f_{x}\right) \frac{\sum_{j \mid E} A_{j}^{u} u_{j}+S_{E}^{u} \Delta V_{E}}{A_{P \mid E}^{u}} \\
\frac{1}{A_{P \mid e}^{u}} & =f_{x} \frac{1}{A_{P \mid P}^{u}}+\left(1-f_{x}\right) \frac{1}{A_{P \mid E}^{u}}
\end{aligned}
$$

$f_{x}$ is a geometric weighting factor with a value of one half if the control volumes are of constant size. Alternatively a harmonic mean may be employed as interpolant. Date [8] argues that the inverse of the diagonal coefficient acts as a diffusion factor in the pressure correction equation (and in the pressure equation, if any) and, as with all diffusion factors, the harmonic mean is more appropriate. The harmonic mean for the inverse is equivalent to calculating an arithmetic mean for the coefficient. If no mention is made the harmonic mean for the inverse is assumed in this work, hence the coefficient at the interface is calculated as $A_{P \mid e}^{u}={\overline{A_{P \mid i}^{u}}}^{e}$. The overline notation represents a mean over the adjacent nodes that share the corresponding interface made explicit at the overline.

$$
\bar{\phi} \cdot \mid i^{e}=f_{x} \phi_{\cdot \mid P}+\left(1-f_{x}\right) \phi_{\cdot \mid E}
$$

$f_{x}$ being a geometric factor. By using Eqs. 1 and 2, Eq. 4 can be written as

$$
\begin{aligned}
u_{e}^{*} & =f_{x} u_{P}^{*}+\left(1-f_{x}\right) u_{E}^{*}+ \\
& +\left.f_{x} \frac{\Delta V_{P}}{A_{P \mid P}^{u}} \frac{\partial p}{\partial x}\right|_{P}+\left.\left(1-f_{x}\right) \frac{\Delta V_{E}}{A_{P \mid E}^{u}} \frac{\partial p}{\partial x}\right|_{P}-\left.\frac{\Delta V_{e}}{A_{P \mid e}^{u}} \frac{\partial p}{\partial x}\right|_{e}= \\
& =\underbrace{\overline{u_{i}^{*}}}_{u_{m}}-\underbrace{\left.\frac{\Delta V_{e}}{A_{P \mid e}^{u}} \frac{\partial p}{\partial x}\right|_{e}+\overline{\left(\left.\frac{\Delta V_{i}}{A_{P \mid i}^{u}} \frac{\partial p}{\partial x}\right|_{i}\right)}=u_{m}+u_{c}}_{u_{c}}
\end{aligned}
$$

$u_{m}$ is the mean over adjacent nodes and $u_{c}$ is the correction related to the pressure field. Date [9] considers that $u_{c}$ can be regarded as a correction that should be zero if the pressure gradient is constant. One of the situations in which this should occur is the fully developed flow in a duct where the velocity associated to $e$ should be the same as those of the nodes, with no contribution from the constant pressure gradient. 
There are some alternatives to the PWIM interpolation, Eq. 7. For instance MWIM would interpolate the $H_{e}^{u}$ term of the $e$ equation given by

$$
H_{e}^{u}=\frac{\sum_{j \mid e} A_{j}^{u} u_{j}^{*}+S_{e}^{u} \Delta V_{e}}{A_{P \mid e}^{u}}
$$

in the following way

$$
\frac{A_{j \mid e}^{u}}{A_{P \mid e}^{u}}=\overline{\left(\frac{A_{j \mid i}^{u}}{A_{P \mid i}^{u}}\right)^{e}} \quad u_{j \mid e}^{*}=\overline{u_{j \mid i}^{*}} e \quad \frac{S_{e}^{u} \Delta V_{e}}{A_{P \mid e}^{u}}=\overline{\left(\frac{S_{i}^{u} \Delta V_{i}}{A_{P \mid i}^{u}}\right)^{e}}
$$

whereas PWIM would use

$$
\begin{aligned}
H_{e}^{u}=\frac{\sum_{j \mid e} A_{j}^{u} u_{j}^{*}+S_{e}^{u} \Delta V_{e}}{A_{P \mid e}^{u}} & =\overline{\left(\frac{\sum_{j \mid i} A_{j}^{u} u_{j}^{*}+S_{i}^{u} \Delta V_{i}}{A_{P \mid i}^{u}}\right)} \\
& =\overline{\left(\frac{\sum_{j \mid i} A_{j}^{u} u_{j}^{*}}{A_{P \mid i}^{u}}\right)^{e}}+\overline{\left(\frac{S_{i}^{u} \Delta V_{i}}{A_{P \mid i}^{u}}\right)^{e}}
\end{aligned}
$$

Comparing Eq. 9 and 10 it can be realized that the only (minor) difference is in the term containing the velocities. Once the solution has converged the values obtained following both techniques are very similar. The convergence rate may slow down slightly with this latter technique if $u_{j \mid e}^{*}$ is calculated with velocity values of the previous iteration as MWIM does not allow to write down an expression like Eq. 7 where the most recent velocity values are used. In fact, $u_{e}^{*}$ can be written as

$$
u_{e}^{*}=H_{e}^{u}-\left.\frac{\Delta V_{e}}{A_{P \mid e}^{u}} \frac{\partial p}{\partial x}\right|_{e}
$$

with $H_{e}^{u}$ calculated from Eqs. 8 and 9 (MWIM) or from Eq. 5 (PWIM). If the MWIM approach uses the momentum factors, $H_{E}^{u}$ and $H_{P}^{u}$, before the equation is solved, the iterative solution will lag behind the one that employs the most recent velocity values. Alternatively, $u_{j \mid e}^{*}$ in Eq. 9 can be estimated as ${\overline{u_{j \mid i}^{*}}}^{e}$, employing the newly available nodal values. Xu and Zhang [10] compared PWIM in both formulations and showed that the one using the most recent values was always faster to converge.

The introduction of underrelaxation is often required to obtain a stable iterative procedure. The equation for $u_{P}$ when an underrelaxation factor $\alpha_{u}$ is incorporated is 


$$
\begin{aligned}
u_{P}^{*} & =\alpha_{u} \frac{\sum_{i \mid P} A_{i}^{u} u_{i}^{*}+S_{P}^{u} \Delta V_{P}}{A_{P \mid P}^{u}}-\left.\alpha_{u} \frac{\Delta V_{P}}{A_{P \mid P}^{u}} \frac{\partial p}{\partial x}\right|_{P}+\left(1-\alpha_{u}\right) u_{P}^{o}= \\
& =\frac{\sum_{i \mid P} A_{i}^{u} u_{i}^{*}+S_{P}^{u} \Delta V_{P}}{\widehat{A}_{P \mid P}^{u}}-\left.\frac{\Delta V_{P}}{\widehat{A}_{P \mid P}^{u}} \frac{\partial p}{\partial x}\right|_{P}+\left(1-\alpha_{u}\right) u_{P}^{o}
\end{aligned}
$$

where the superindex ' $O$ ' refers to the previous iteration. An equivalent expression can be written for $u_{E}$. The equations are identical to the nonrelaxed ones if $A^{u}$ is substituted by $\widehat{A}^{u}=A^{u} / \alpha_{u}$ and the contribution from the previous iteration is included. Operating in the same manner as before one obtains

$$
\begin{aligned}
& u_{e}^{*}={\widehat{\widehat{H}_{i}^{u}}}^{e}-\left.\frac{\Delta V_{e}}{\widehat{A}_{P \mid e}^{u}} \frac{\partial p}{\partial x}\right|_{e}+\left(1-\alpha_{u}\right) u_{e}^{o}= \\
& ={\overline{u_{i}^{*}}}^{e}+\overline{\left(\left.\frac{\Delta V_{i}}{\widehat{A}_{P \mid i}^{u}} \frac{\partial p}{\partial x}\right|_{i}\right)^{e}-\left.\frac{\Delta V_{e}}{\widehat{A}_{P \mid e}^{u}} \frac{\partial p}{\partial x}\right|_{e}+\left(1-\alpha_{u}\right)\left[u_{e}^{o}-\overline{u_{i}^{o}}\right]}
\end{aligned}
$$

The above equation provides a solution independent of the underelaxation factor as

$$
u_{e}^{*}={\overline{u_{i}^{*}}}^{e}+\alpha_{u} u_{c}+\left(1-\alpha_{u}\right) u_{c}^{o}
$$

$u_{c}$ and $u_{c}^{o}$ being the pressure field contributions at the current and previous iterations given by

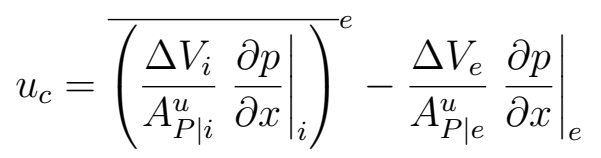

Note that $A^{u}$ does not contain the underrelaxation factor. When the final solution is reached $u_{c}=u_{c}^{o}$ and $u_{e}^{*}=u_{m}+u_{c}$. Eq. 13 highlights the importance of carrying out a proper interpolation when calculating the velocity at $e$. If $\left(1-\alpha_{u}\right) u_{e}^{o}$ is included in $H_{e}^{u}$ and this term interpolated as Eq. 5 indicates, the final expression is

$$
\begin{aligned}
u_{e}^{*} & ={\overline{u_{i}^{*}}}^{e}+\overline{\left(\left.\frac{\Delta V_{i}}{\widehat{A}_{P \mid i}^{u}} \frac{\partial p}{\partial x}\right|_{i}\right)^{e}}-\left.\frac{\Delta V_{e}}{\widehat{A}_{P \mid e}^{u}} \frac{\partial p}{\partial x}\right|_{e}= \\
& ={\overline{u_{i}^{*}}}^{e}+\alpha_{u}\left[\overline{\left(\left.\frac{\Delta V_{i}}{A_{P \mid i}^{u}} \frac{\partial p}{\partial x}\right|_{i}\right)}-\left.\frac{\Delta V_{e}}{A_{P \mid e}^{u}} \frac{\partial p}{\partial x}\right|_{e}\right]= \\
& ={\overline{u_{i}^{*}}}^{e}+\alpha_{u} u_{c}
\end{aligned}
$$

which shows that the value would depend on the relaxation factor. Majumdar [2] was the first to show the interpolation leading to Eq. 13 although he 
followed a path slightly different from the one taken here. The requirement that the contribution from the previous iteration should not be included in $H_{e}^{u}$ in order to have a solution independent of the relaxation factor was first observed by Miller and Schmidt [3] and later employed by others [31].

The $u_{e}^{*}$ expression in Eq. 13 considers $H_{e}^{u}$ in Eq. 5 as the variable to be interpolated but an alternative expression can be derived if the interpolation is performed over $\sum_{j \mid e} A_{j}^{u} u_{j}^{*}+S_{e}^{u}$. In this case Eq. 13 transforms into

$$
\begin{aligned}
u_{e}^{*} & =\frac{1}{A_{P \mid e}^{u}} \overline{A_{P \mid i}^{u} u_{i}^{*}}+\alpha_{u} \frac{1}{A_{P \mid e}^{u}}\left(\overline{\left.\Delta V_{i} \frac{\partial p}{\partial x}\right|_{i} ^{e}}-\left.\Delta V_{e} \frac{\partial p}{\partial x}\right|_{e}\right)+ \\
& +\left(1-\alpha_{u}\right)\left(u_{e}^{o}-\frac{1}{A_{P \mid e}^{u}} \overline{A_{P \mid i}^{u} u_{i}^{o}}\right)
\end{aligned}
$$

Many researchers [14] [15] [18] employ a similar expression but others do not consider the ratio between the coefficients $\left(A_{P \mid i}^{u}\right.$ and $\left.A_{P \mid e}^{u}\right)$ that comes about when performing a correct weighting procedure [19] [20] [21] [22] [23], that is, they employ the pressure term as in Eq. 17 but the other terms are as in Eq. 13. Often, the reason behind the use of a pressure term in this fashion is just convenience. If the term is computed in that form the pressure contributes to the cell mass balance with a strict (dissipative) fourth order derivative, as opposed to the third order derivative of the other form due to the (not always slight) variation of $A_{P \mid i}^{u}$. The amount of dissipation is sometimes controlled by a coefficient (other than the geometric factor) that is adjusted ad hoc [19] [20] [24]. In the computational exercices to be presented with the unsteady version of this scheme we prefer to be consistent and stick to the correct expression given in Eq. 17. The coefficients in the $u_{m}$ term should not be derived independently from those of the pressure field term, $u_{c}$.

\section{SIMPLE, SIMPLEC and SIMPLER in a colocated grid. Steady case}

As it is important to realize the whole implications of the proposals employed in the past, a discussion of the hypothesis behind the expression that links the nodal and face velocity corrections to the pressure correction gradients is developed in this section. SIMPLE, SIMPLEC and SIMPLER are the three procedures usually employed in a structured colocated grid and all three will be described next. We will observe that in order to produce a consistent result there is also a restriction in the way we define the pseudovelocities in the pressure equation in SIMPLER. 
Let $u_{P}^{n}$ be the velocity at the previous iteration, $u_{P}^{*}$ be the velocity right after calculating the momentum equations and $u_{P}^{n+1}$ be the velocity at the next iteration after improving $u_{P}^{*}$ based on continuity satisfaction, all of them at node $P$. The equation for $u_{P}^{*}$ is

$$
u_{P}^{*}=\widehat{H}_{P}^{*}-\left.\frac{\Delta V_{P}}{\widehat{A}_{P \mid P}^{u}} \frac{\partial p}{\partial x}\right|_{P} ^{n}+\left(1-\alpha_{u}\right) u_{P}^{n}
$$

The momentum equation associated to the velocities that satify continuity is

$$
u_{P}^{n+1}=\widehat{H}_{P}^{n+1}-\left.\frac{\Delta V_{P}}{\widehat{A}_{P \mid P}^{u}} \frac{\partial p}{\partial x}\right|_{P} ^{n+1}+\left(1-\alpha_{u}\right) u_{P}^{n}
$$

Subtracting Eq. 18 from Eq. 19 we obtain the equation for the velocity corrections

$$
u_{P}^{\prime}=\widehat{H}_{P}^{\prime}-\left.\frac{\Delta V_{P}}{\widehat{A}_{P \mid P}^{u}} \frac{\partial p}{\partial x}\right|_{e} ^{\prime}
$$

where the variation of $\widehat{A}_{P \mid P}^{u}$ is neglected. SIMPLE adopts a series of simplifications, in particular neglects $H^{\prime}$ (SIMPLE does but SIMPLEC does not, at least partially) and ends up with a very convenient equation

$$
u_{P}^{\prime}=-\left.\frac{\Delta V_{P}}{\widehat{A}_{P \mid P}^{u}} \frac{\partial p}{\partial x}\right|_{P} ^{\prime}
$$

The cell face velocity is given by

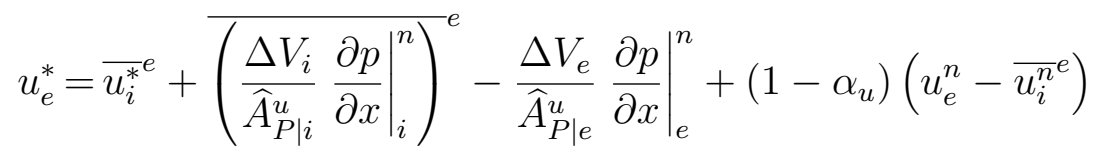

And the corresponding equation for the convecting velocity that satisfies continuity is

$$
u_{e}^{n+1}={\overline{u_{i}^{n+1}}}^{e}+\overline{\left(\left.\frac{\Delta V_{i}}{\widehat{A}_{P \mid i}^{u}} \frac{\partial p}{\partial x}\right|_{i} ^{n+1}\right)^{e}}-\left.\frac{\Delta V_{e}}{\widehat{A}_{P \mid e}^{u}} \frac{\partial p}{\partial x}\right|_{e} ^{n+1}+\left(1-\alpha_{u}\right)\left(u_{e}^{n}-{\overline{u_{i}^{n}}}^{e}\right)
$$

Subtracting Eq. 22 from Eq. 23

$$
u_{e}^{\prime}=\overline{u_{i}^{\prime}}{ }^{e}+\overline{\left(\left.\frac{\Delta V_{i}}{\widehat{A}_{P \mid i}^{u}} \frac{\partial p}{\partial x}\right|_{i} ^{\prime}\right)^{e}}-\left.\frac{\Delta V_{e}}{\widehat{A}_{P \mid e}^{u}} \frac{\partial p}{\partial x}\right|_{e} ^{\prime}
$$


and

$$
u_{e}^{\prime}=-\left.\frac{\Delta V_{e}}{\widehat{A}_{P \mid e}^{u}} \frac{\partial p}{\partial x}\right|_{e} ^{\prime}
$$

as, due to Eq. 21,

$$
{\overline{u_{i}^{\prime}}}^{e}=-\overline{\left(\left.\frac{\Delta V_{i}}{\widehat{A}_{P \mid i}^{u}} \frac{\partial p}{\partial x}\right|_{i} ^{\prime}\right)^{e}}
$$

The relation between the face velocity correction and the pressure correction gradient is formally identical to that of the nodal velocities.

SIMPLEC requires a bit more algebra because the simplifications adopted give rise to two different expressions if SIMPLEC idea is applied to both the face velocities and the nodal velocities or it is only applied to the nodal velocities and the face expression is derived from these. The option followed in this work is the first one that provides equivalent equations to those of SIMPLE, Eqns. 21 and 26.

$$
u_{P}^{\prime}=-\left.\frac{\Delta V_{P}}{\breve{A}_{P \mid P}^{u}} \frac{\partial p}{\partial x}\right|_{P} ^{\prime} \quad \text { and } \quad u_{e}^{\prime}=-\left.\frac{\Delta V_{e}}{\breve{A}_{P \mid e}^{u}} \frac{\partial p}{\partial x}\right|_{e} ^{\prime}
$$

with $\breve{A}=\widehat{A}-\sum_{j} A_{j}$. To obtain the pressure correction equation the discretized continuity equation for the node $P$ at the $n+1$ iteration is constructed, in which $u^{n+1}=u^{*}+u^{\prime}$. The assembling of the interface velocity expressions and the substitution of $u_{e}^{\prime}$ and the like via Eq. 25 and similar ones finally produces the equation sought.

The pressure equation for SIMPLER starts with the equation for $u_{e}^{n+1}$, velocity that we consider satisfies continuity.

$$
u_{e}^{n+1}=\widehat{H}_{e}^{*}-\left.\frac{\Delta V_{e}}{\widehat{A}_{P \mid e}^{u}} \frac{\partial p}{\partial x}\right|_{e} ^{n+1}+\left(1-\alpha_{u}\right) u_{e}^{n}
$$

and calculates $\widehat{H}_{e}^{*}={\widehat{\widehat{H}_{i}^{*}}}^{e}$. SIMPLER then looks for the unknown pressure field at iteration $n+1$ that on being inserted in the momentum equation makes the resulting velocity field satisfy continuity. That reduces to substituting the RHS of the previous equation and the rest of face values at the $n+1$ step in the continuity equation. After a little algebra it can be shown that the coefficients are the same as in the pressure correction equation, the only difference being the source term. We stress again that incorporating $\left(1-\alpha_{u}\right) u_{e}^{n}$ in the $H_{e}^{*}$ term 
to be interpolated (the pseudovelocity) causes the solution to be inconsistent, i.e., dependent on the relaxation factor.

\section{The interface velocity in an unsteady flow}

Let us consider now the case of an unsteady flow. We will present a new expression for $u_{e}^{*}$ and will relate it to previous ones in the literature. We will proceed in a similar fashion to the steady case by first writing the fictitious momentum equation at interface $e$ for a variable density case

$$
\begin{aligned}
A_{P \mid e}^{u} u_{e}^{*} & =\sum_{j \mid e} A_{j}^{u} u_{j}^{*}+S_{e}^{u} \Delta V_{e}-\left.\Delta V_{e} \frac{\partial p}{\partial x}\right|_{e} ^{l}+ \\
& +\frac{1-\alpha_{u}}{\alpha_{u}} \widetilde{A}_{P \mid e}^{u}\left(u_{e}^{l}-u_{e}^{*}\right)+\frac{\rho_{e} \Delta V_{e}}{\Delta t}\left(u_{e}^{n}-u_{e}^{*}\right)
\end{aligned}
$$

with

$$
\widetilde{A}_{P \mid e}^{u}=A_{P \mid e}^{u}+\frac{\rho_{e} \Delta V_{e}}{\Delta t} \quad A_{P \mid e}^{u}=\sum_{j \mid e} A_{j}^{u}
$$

The most general case in which a (real or pseudo) time step can be combined with underrelaxation, that may also be interpreted as a local pseudotime step, will be described. By letting $\Delta t \rightarrow \infty$ the underrelaxed equation is obtained and if $\alpha_{u}$ is equal to one, time marching is used to seek the steady state. The equation is written in this way to quickly identify the meaning of the terms. The second to last term on the right hand side is the contribution of the change in $u_{e}^{*}$ from (relaxed) iteration to (relaxed) iteration within a given time step, and the last term is the contribution of the unsteady change. The $u_{e}^{l}$ is the value at the previous inner iteration and $u_{e}^{n}$ is the value at the preceding time step. The algebraic equation is cast in this form because it becomes obvious that when there is no change in $u_{e}$, that is, $u_{e}^{*}=u_{e}^{l}=u_{e}^{n}$, it reduces to the discretized steady equation with no relaxation, so the final (steady) solution will not depend on either $\alpha_{u}$ or $\Delta t$. There has been much controversy over the years as to what is the expression for $u_{e}^{*}$ that is independent of these two parameters. Starting from this equation we will be able to derive a correct expression for $u_{e}^{*}$ and to spot the (sometimes subtle) errors in other expressions put forward by previous researchers. The equation for the node $\mathrm{P}$ that shares interface $e$ is

$$
A_{P \mid P}^{u} u_{P}^{*}=\sum_{j \mid P} A_{j}^{u} u_{j}^{*}+S_{P}^{u} \Delta V_{P}-\left.\Delta V_{P} \frac{\partial p}{\partial x}\right|_{P} ^{l}+
$$




$$
+\frac{1-\alpha_{u}}{\alpha_{u}} \widetilde{A}_{P \mid P}^{u}\left(u_{P}^{l}-u_{P}^{*}\right)+\frac{\rho_{P} \Delta V_{P}}{\Delta t}\left(u_{P}^{n}-u_{P}^{*}\right)
$$

Following the notation introduced before, the original Rhie-Chow interpolation assumes that

$$
H_{e}^{u}=\frac{\sum_{j \mid e} A_{j}^{u} u_{j}^{*}+S_{e}^{u} \Delta V_{e}}{A_{P \mid e}^{u}}=\overline{\left(\frac{\sum_{j \mid i} A_{j}^{u} u_{j}^{*}+S_{i}^{u} \Delta V_{i}}{A_{P \mid i}^{u}}\right)}={\overline{H_{i}^{u}}}^{e}
$$

wich can be interpreted as an assumption of linearity for $H^{u}$. If we perform this average with the first term of Eq. 31 and the corresponding one of the $u_{E}$ equation, we will be able to express $H_{e}^{u}$ in terms of the rest and the equation for $u_{e}^{*}$ can be written as

$$
\begin{aligned}
& u_{e}^{*}=\overline{u_{i}^{*}}+\overline{\left.\frac{\Delta V_{i}}{A_{P \mid i}^{u}} \frac{\partial p}{\partial x}\right|_{i} ^{l}}-\left.\frac{\Delta V_{e}}{A_{P \mid e}^{u}} \frac{\partial p}{\partial x}\right|_{e} ^{l}+ \\
& +\frac{1-\alpha_{u}}{\alpha_{u}} \frac{\widetilde{A}_{P \mid e}^{u}}{A_{P \mid e}^{u}}\left(u_{e}^{l}-u_{e}^{*}\right)-{\overline{\frac{1-\alpha_{u}}{\alpha_{u}}} \frac{\widetilde{A}_{P \mid i}^{u}}{A_{P \mid i}^{u}}\left(u_{i}^{l}-u_{i}^{*}\right)}^{e}+ \\
& +\frac{\rho_{e} \Delta V_{e}}{\Delta t A_{P \mid e}^{u}}\left(u_{e}^{n}-u_{e}^{*}\right)-\overline{{\overline{\rho_{i} \Delta V_{i}}}_{\Delta t A_{P \mid i}^{u}}\left(u_{i}^{n}-u_{i}^{*}\right)}
\end{aligned}
$$

This is not the equation to be implemented because $u_{e}^{*}$ is in both sides but written in this way one can grasp the independency of the final solution from both the underrelaxation factor and the time step. The first line on the RHS, that it is the only one remaining in the final solution, is the steady state unrelaxed solution. Note that the $A_{P \mid i}^{u}$ factors in the pressure gradient do not contain the unsteady contribution so when $u_{e}^{*}=u_{e}^{l}=u_{e}^{n}$ the final solution is the steady unrelaxed solution, as it should be. Reordering the equation one obtains

$$
\begin{aligned}
\left(1+\delta_{e}\right) u_{e}^{*} & =\overline{\left(1+\delta_{i}\right) u_{i}^{*}}+\alpha_{u} \Delta t\left[\overline{\left.\frac{\delta_{i}}{\rho_{i}} \frac{\partial p}{\partial x}\right|_{i} ^{l}}-\left.\frac{\delta_{e}}{\rho_{e}} \frac{\partial p}{\partial x}\right|_{e} ^{l}\right]+ \\
& +\left(1-\alpha_{u}\right)\left[\left(1+\delta_{e}\right) u_{e}^{l}-\overline{\left(1+\delta_{i}\right) u_{i}^{l}}\right]+\alpha_{u}\left[\delta_{e} u_{e}^{n}-{\overline{\delta_{i} u_{i}^{n}}}^{e}\right]
\end{aligned}
$$

with

$$
\delta_{e, i}=\frac{\rho_{e, i} \Delta V_{e, i}}{\Delta t A_{P \mid e, i}^{u}}
$$

which is the equation to be coded. This is the equivalent unsteady expression of the Rhie-Chow procedure. When $\Delta t \rightarrow \infty$, that is, $\delta_{e, i} \rightarrow 0$, the Rhie-Chow 
expression, Eqn 13, is recovered. This new expression bears strong resemblance to the one presented by Choi [11], but differs in some very important aspects that, unlike Choi's, make it truly independent of the time step. We named this cell face velocity evaluation as PICTURE (Proper Interpolation for a Colocated Treatment of the Unsteady Reynolds-averaged Equations) because we initially employed it in a (U)RANS code. There are some prior schemes in the literature whose formulation is very close to that just presented. Lai et al. [12] followed a similar approach by averaging the same term as PICTURE but they late adopted a series of simplifications that made the final expression move away from that of PICTURE. The actual $u_{e}^{*}$ equation they employed contains some unnecessary approximations that can produce a less accurate solution. Barton et al. [19] did not derive a special interpolation procedure for $u_{e}^{*}$, however they eventually assemble a continuity equation for an unsteady problem that contains factors like $\Delta V_{i} / A_{P \mid i}^{u}$ and not $\Delta V_{i} / \widetilde{A}_{P \mid i}^{u}{ }^{2}$. As shown later these coefficients multiply the pressure gradient correction in our continuity equation derived from Eqn. 33 so in that sense both schemes end up with a somehow similar continuity equation. The approach taken by Barton et al. is however so different that it cannot be considered a scheme close to PICTURE. Cubero et al. [13] proposed a consistent scheme separating the temporal term from the underrelaxed one, i.e., they did not underrelax the complete $\widetilde{A}_{P \mid P}^{u}=A_{P \mid P}^{u}+\rho \Delta V / \Delta t$ but only $A_{P \mid P}^{u}$, whereas PICTURE works with the underrelaxed $\widetilde{A}_{P \mid P}^{u}$. Apart from this slight change both formulations are almost identical.

The writing of Eq. 29 is not unique and another variant can lead to a different expression for $u_{e}^{*}$, in fact the one proposed by Choi. This non-uniqueness is due to the fact that at some point of the derivation one has to perform an averaging procedure equivalent to the Rhie-Chow proposal over a quantity that is dependent on the form of the starting equation. As the $u_{e}$ momentum equation is fictitious, the way we relate it to the actually solved $u_{E}$ and $u_{P}$ momentum equations is, to some extent, a matter of taste, something that will become clear further on. In this process of connecting terms of a fictitious equation with their counterparts in the ones solved, one important thing to care about is the independence of the steady solution from the time step. We will see that a seemingly correct interpolation, like Choi's, may induce time step dependency.

Let us write the fictitious $u_{e}$ momentum equation in a more traditional fashion

$$
\frac{\widetilde{A}_{P \mid e}^{u}}{\alpha_{u}} u_{e}^{*}=\sum_{j \mid e} A_{j}^{u} u_{j}^{*}+S_{e}^{u} \Delta V_{e}-\left.\Delta V_{e} \frac{\partial p}{\partial x}\right|_{e} ^{l}+\frac{1-\alpha_{u}}{\alpha_{u}} \widetilde{A}_{P \mid e}^{u} u_{e}^{l}+\frac{\rho_{e} \Delta V_{e}}{\Delta t} u_{e}^{n}
$$

$\overline{2}$ The fact that they do not include the temporal terms in the coefficients is explicitly mentioned in the paper 
and

$$
\begin{aligned}
u_{e}^{*} & =\alpha_{u}\left[\frac{\sum_{j \mid e} A_{j}^{u} u_{j}^{*}+S_{e}^{u} \Delta V_{e}}{\widetilde{A}_{P \mid e}^{u}}\right]-\left.\alpha_{u} \frac{\Delta V_{e}}{\widetilde{A}_{P \mid e}^{u}} \frac{\partial p}{\partial x}\right|_{e} ^{l}+\left(1-\alpha_{u}\right) u_{e}^{l}+ \\
& +\alpha_{u} \frac{\delta_{e}}{1+\delta_{e}} u_{e}^{n}
\end{aligned}
$$

The term in brackets is approximated as

$$
\frac{\sum_{j \mid e} A_{j}^{u} u_{j}^{*}+S_{e}^{u} \Delta V_{e}}{\widetilde{A}_{P \mid e}^{u}}={\overline{\left(\frac{\sum_{j \mid i} A_{j}^{u} u_{j}^{*}+S_{i}^{u} \Delta V_{i}}{\widetilde{A}_{P \mid i}^{u}}\right)^{e}}}^{e}
$$

The denominators of Eqs. 32 and 38 are different and the hypothesis of linear variation of the former does not take us to the same point as a similar hypothesis for the latter. The result will depend on the approach taken. Apparently both interpolations are correct $^{3}$ as the only assumption involved in their derivation is the piecewise linearity of the interpolated functions between nodes. Nevertheless, we will show that the second one gives rise to an inconsistent steady solution unless special care is taken in the election of the interpolation operators. This problem did not arise in the steady relaxed equation because the difference between the denominators in the nonrelaxed and relaxed equations was a multiplicative constant for the whole field: $\alpha_{u}$. The case is different now.

Following the same path as before we end up with

$$
\begin{aligned}
u_{e}^{*} & =\overline{u_{i}^{*}}+\alpha_{u} \Delta t\left[\overline{\left.\frac{\delta_{i}}{\rho_{i}\left(1+\delta_{i}\right)} \frac{\partial p}{\partial x}\right|_{i} ^{l}}-\left.\frac{\delta_{e}}{\rho_{e}\left(1+\delta_{e}\right)} \frac{\partial p}{\partial x}\right|_{e} ^{l}\right]+ \\
& +\left(1-\alpha_{u}\right)\left[u_{e}^{l}-{\overline{u_{i}^{l}}}^{e}\right]+\alpha_{u}\left[\frac{\delta_{e}}{1+\delta_{e}} u_{e}^{n}-\frac{\delta_{i}}{1+\delta_{i}} u_{i}^{n}\right]
\end{aligned}
$$

This is in fact the scheme proposed by Choi [11] who claimed it was independent of the time step. The dividing function for Choi's scheme in Eq. 38 is $\widetilde{A}_{P \mid e, i}^{u}$ whereas in PICTURE is $A_{P \mid e, i}^{u}$, that is why every term in this equation is $1 /(1+\delta)$ times the corresponding one in PICTURE. Observe that the asymptotic behaviour is correct, if $\Delta t \rightarrow \infty$ Rhie-Chow expression is obtained. The inconsistency of this scheme arises for small time steps and quickly worsens as the time step goes to zero. Yu et al. [17] [18] pointed out that this approach produced a steady state solution that was $\Delta \mathrm{t}$-dependent by showing the variation of the final value at monitoring points for different $\Delta t$ in a lid-driven

$\overline{3}$ Not neccesarily of equal accuracy 
cavity case. Their numerical findings were supported by an algebraic derivation which concluded that in fact Choi's scheme was time step dependent. Its assertion will be qualified in this work following a simpler theoretical analysis and will be underpinned by the computational evidence. Later we will show that Choi's extension to unsteady flows can be made time step independent with a judicious choice of the interpolation operator. We postpone a lengthier discussion on this issue to a next section, now we will explain another interpolation practice in which the election of the starting equation is irrelevant.

If the assumption of linearity is taken over $\sum_{i \mid e} A_{i}^{u} u_{i}^{*}+S_{e}^{u}$ instead of the function given in Eq. 38, the equation obtained is

$$
\begin{aligned}
A_{P \mid e}^{u} u_{e}^{*} & =\overline{A_{P \mid i}^{u} u_{i}^{*}}+\left[\overline{\left.\Delta V_{i} \frac{\partial p}{\partial x}\right|_{i} ^{l}}-\left.\Delta V_{e} \frac{\partial p}{\partial x}\right|_{e} ^{l}\right]+ \\
& +\frac{1-\alpha_{u}}{\alpha_{u}} \widetilde{A}_{P \mid e}^{u}\left(u_{e}^{l}-u_{e}^{*}\right)-\frac{1-\alpha_{u}}{\alpha_{u}} \widetilde{A}_{P \mid i}^{u}\left(u_{i}^{l}-u_{i}^{*}\right)^{e}+ \\
& +\frac{\rho_{e} \Delta V_{e}}{\Delta t}\left(u_{e}^{n}-u_{e}^{*}\right)-\frac{\rho_{i} \Delta V_{i}}{\Delta t}\left(u_{i}^{n}-u_{i}^{*}\right)
\end{aligned}
$$

which can be written in an alternative way as

$$
\begin{aligned}
& \frac{1}{\alpha_{u}} \widetilde{A}_{P \mid e}^{u} u_{e}^{*}=\frac{1}{\alpha_{u}}{\widetilde{A_{P \mid i}^{u} u_{i}^{*}}}^{e}+\left[\overline{\left.\Delta V_{i} \frac{\partial p}{\partial x}\right|_{i} ^{l}}-\left.\Delta V_{e} \frac{\partial p}{\partial x}\right|_{e} ^{l}\right]^{e}+ \\
& +\frac{1-\alpha_{u}}{\alpha_{u}}\left(\widetilde{A}_{P \mid e}^{u} u_{e}^{l}-{\widetilde{A_{P \mid i}^{u} u_{i}^{l}}}^{e}\right)+ \\
& +\frac{\rho_{e} \Delta V_{e}}{\Delta t} u_{e}^{n}-\frac{\overline{\rho_{i} \Delta V_{i}}}{\Delta t} u_{i}^{n}
\end{aligned}
$$

or

$$
\begin{aligned}
A_{P \mid e}^{u}\left(1+\delta_{e}\right) u_{e}^{*} & =\overline{A_{P \mid i}^{u}\left(1+\delta_{i}\right) u_{i}^{*}}{ }^{e}+\alpha_{u}\left[\overline{\left.\Delta V_{i} \frac{\partial p}{\partial x}\right|_{i} ^{l}}-\left.\Delta V_{e} \frac{\partial p}{\partial x}\right|_{e} ^{l}\right]+ \\
& +\left(1-\alpha_{u}\right)\left(A_{P \mid e}^{u}\left(1+\delta_{e}\right) u_{e}^{l}-\overline{A_{P \mid i}^{u}\left(1+\delta_{i}\right) u_{i}^{l}} e^{e}\right)+ \\
& +\alpha_{u}\left(A_{P \mid e}^{u} \delta_{e} u_{e}^{n}-\overline{A_{P \mid i}^{u} \delta_{i} u_{i}^{n}}{ }^{e}\right.
\end{aligned}
$$

This is the form originally employed by Kadja et al. [14] [15] and Udaykumar et al. [16], and later claimed by $\mathrm{Yu}$ et al [17] to be the only form that is unconditionally free of $\Delta$ t-dependence. The new interpolation presented in Eq. 34 also shares this property, something that will be demonstrated later. In all the derivations presented the source term, other than the pressure gradient and the contribution from previous values, is included in the term to 
be interpolated. In some instances, for example when dealing with strongly buoyant flows, it may be convenient to treat it separately to obtain physically reasonable solutions [25] [26] [27], although other researchers have not found it necessary [7]. Also, when the pressure gradient and the source term have to be exactly compensated in both the cell volume and that of the face velocity an identical treatment is also required [28] and that implies keeping the source term in the same brackets as the pressure gradient. As an example, with this latter approach PICTURE expression would be

$$
\begin{aligned}
\left(1+\delta_{e}\right) u_{e}^{*} & =\overline{\left(1+\delta_{i}\right) u_{i}^{*}}+\alpha_{u} \Delta t\left[\overline{\delta_{i}\left(\left.\frac{\partial p}{\rho_{i}}\right|^{l}-S_{i}^{u}\right)^{e}}-\frac{\delta_{e}}{\rho_{e}}\left(\left.\frac{\partial p}{\partial x}\right|_{e} ^{l}-S_{e}^{u}\right)\right]+ \\
& +\left(1-\alpha_{u}\right)\left[\left(1+\delta_{e}\right) u_{e}^{l}-\overline{\left(1+\delta_{i}\right) u_{i}^{e}}\right]+ \\
& +\alpha_{u}\left[\delta_{e} u_{e}^{n}-{\overline{\delta_{i} u_{i}^{n}}}^{e}\right]
\end{aligned}
$$

It is worth mentioning that there have been other attempts to circumvent the problem of a nonunique solution linked to an inconsistent behaviour as the time step goes to zero or to infinity, as in Shen et al. [29] [30]. In these papers the authors included the contribution of the values of the previous time intervals in the $H_{e}$ interpolation when assembling the primary $u_{e}$ equation, something that is not correct. They were more interested in getting rid of some wiggles in the solution and put forward another expression for $u_{e}$ (actually for the convective flux) that allowed them to obtain a smooth solution. Nevertheless, they still produced a time step dependent solution (however slight this dependency) as can be spotted in some of the figures in the paper. Its investigation is important in itself because it shows that sometimes another (undesirable) side effect of not carrying out a proper interpolation is the appearance of saw-tooth unphysical profiles. Nevertheless, this is not a problem exclusive of inconsistent interpolations as some (smaller amplitude) wiggles can also appear in the solution with consistent interpolations, especially in regions of large pressure variations.

In the second paper [30] an alternative way of writing the $u_{e}$ equation is proposed. Its starting point is Eq. 29 rewritten in the following manner

$$
\begin{aligned}
\frac{\rho_{e} \Delta V_{e}}{\Delta t} u_{e}^{*} & =\sum_{j \mid e} A_{j}^{u} u_{j}^{*}+S_{e}^{u} \Delta V_{e}-\left.\Delta V_{e} \frac{\partial p}{\partial x}\right|_{e} ^{l}+ \\
& +\frac{1-\alpha_{u}}{\alpha_{u}} \widetilde{A}_{P \mid e}^{u}\left(u_{e}^{l}-u_{e}^{*}\right)+\frac{\rho_{e} \Delta V_{e}}{\Delta t} u_{e}^{n}-A_{P \mid e}^{u} u_{e}^{*}
\end{aligned}
$$

which is adapted from the one in the paper to conform to our notation. Note that it is the temporal contribution what is kept on the LHS. Shen et al. 
presented this approach within a SIMPLEC procedure, although it can be derived with no specification of the PV coupling scheme adopted. Now the interpolation is realized over

$$
\frac{\sum_{j \mid e} A_{j}^{u} u_{j}^{*}+S_{e}^{u} \Delta V_{e}}{\rho_{e} \Delta V_{e}} \Delta t=\Delta t{\overline{\left(\frac{\sum_{j \mid i} A_{j}^{u} u_{j}^{*}+S_{i}^{u} \Delta V_{i}}{\rho_{i} \Delta V_{i}}\right)}}^{e}
$$

Because of the error mentioned Shen et al had to introduce a new $\beta$ factor to make it $\Delta \mathrm{t}$ independent but here we will not pursue his approach, instead we will follow our procedure to see if we can obtain a proper $\Delta$ t-independent scheme. After a little algebra the $u_{e}$ equation may be written as

$$
\begin{aligned}
\frac{1+\delta_{e}}{\delta_{e}} u_{e}^{*} & =\frac{\overline{1+\delta_{i}}}{\delta_{i}} u_{i}^{*}+\alpha_{u} \Delta t\left[\left.\frac{1}{\rho_{e}} \frac{\partial p}{\partial x}\right|_{e} ^{l}-\overline{\left.\frac{1}{\rho_{i}} \frac{\partial p}{\partial x}\right|_{i} ^{l}}\right]+ \\
& +\left(1-\alpha_{u}\right)\left[\frac{1+\delta_{e}}{\delta_{e}} u_{e}^{l}-\frac{\overline{1+\delta_{i}}}{\delta_{i}} u_{i}^{l}\right]+ \\
& +\alpha_{u}\left(u_{e}^{n}-\overline{u_{i}^{n} e}\right)
\end{aligned}
$$

scheme that provides a final solution that is also independent of $\Delta \mathrm{t}$. This scheme will be named PICTURETWO (PICTURE with a Tricky Weighted Operand). The tricky weighted operand refers to Eq. 45. It is straightforward to show that this scheme is equivalent to Kadja et al's if the cell volume is constant over the domain $\left(\Delta V_{e}=\Delta V_{E}=\Delta V_{P}\right)$. Although this scheme will be shown to be only conditionally consistent, it will serve us as an example of how the criterium for unconditional $\Delta \mathrm{t}$ independency proposed in this paper can detect other dependencies not inmediately obvious.

When implementing the unsteady procedure SIMPLE and SIMPLEC expressions are the same as in the relaxed case if we substitute $A$ by $\widetilde{A}$. SIMPLER contains a little more terms on the RHS. For instance for the new consistent interpolation presented in this paper $u_{e}^{n+1}$ can be written as

$$
u_{e}^{n+1}=\frac{\alpha_{u}}{1+\delta_{e}} \bar{H}_{i}^{e}-\left.\alpha_{u} \frac{\Delta t}{\rho_{e}} \frac{\delta_{e}}{1+\delta_{e}} \frac{\partial p}{\partial x}\right|_{e} ^{n+1}+\left(1-\alpha_{u}\right) u_{e}^{l}+\alpha_{u} \frac{\delta_{e}}{1+\delta_{e}} u_{e}^{n}
$$

with $H$ given in Eq. 32. Again the coefficients are the same in the pressure and the pressure correction equations.

If only the steady state is sought there is an extremely radical approach: to neglect all terms that represent the net changes from previous iterations in the $u_{e}^{*}$ expression, both relaxation-related and time-related. That amounts to only keeping the first two lines of the RHS of Eq. 33 in which there is no contribution from previous values. By doing so the computational cost in terms 
of storage needs is reduced and no $\alpha_{u}$ and $\Delta \mathrm{t}$ is present. This drastic simplification involves neglecting terms which can be of significance and hence the convergence deteriorates (or the procedure does not converge at all) in much the same way as when neglecting neighbour velocity corrections in SIMPLElike procedures. Although its limitations, this approach has been followed by some researchers [20].

\section{Alternatives for interpolating the coefficients}

This section explores and discusses different alternatives for the calculation of cell face coefficients that appear in the cell face velocity expression. The alternatives appear because the $u_{e}$ equation is fictitious and hence the expression that links its coefficients with those in the equations solved (for $u_{E}$ and $\left.u_{P}\right)$ is not unique. In particular the discussion will be focused on the $\widetilde{A}_{P \mid e}^{u}$ coefficient. As mentioned previously we consider a weighted arithmetic mean as the standard value.

$$
\widetilde{A}_{P \mid e}^{u}={\widetilde{\widetilde{A}_{P \mid i}^{u}}}^{e}
$$

Let us consider the formal expression

$$
\widetilde{A}_{P \mid e}^{u}=A_{P \mid e}^{u}+\frac{\rho_{e} \Delta V_{e}}{\Delta t}=A_{P \mid e}^{u}+\frac{\Delta M_{e}}{\Delta t}
$$

$\Delta M_{e}$ being the mass contained in the e-volume. We can write

$$
{\widetilde{A_{P \mid i}^{u}}}^{e}={\overline{A_{P \mid i}^{u}}}^{e}+\frac{\Delta M_{e}}{\Delta t}
$$

Following Date [8] a weighted arithmetic mean with a $f_{x}$ geometric factor will be employed allowing for variable cell sizes. Developing Eq. 48 we have

$$
\begin{aligned}
{\widetilde{\widetilde{A}_{P \mid i}^{u}}}^{e} & =f_{x} \widetilde{A}_{P \mid P}^{u}+\left(1-f_{x}\right) \widetilde{A}_{P \mid E}^{u}= \\
& =f_{x} A_{P \mid P}^{u}+\left(1-f_{x}\right) A_{P \mid E}^{u}+\frac{1}{\Delta t}\left(f_{x} \Delta M_{P}+\left(1-f_{x}\right) \Delta M_{E}\right)
\end{aligned}
$$

expression that is incompatible with Eq. 50 as in general $\Delta M_{e} \neq f_{x} \Delta M_{P}+$ $\left(1-f_{x}\right) \Delta M_{E}$, only it is true in a uniform grid. In fact, in a nonuniform grid the mass contained in the e-volume is $\Delta M_{e}=\widetilde{f}_{x}\left(\Delta M_{P}+\Delta M_{E}\right)$ with $\widetilde{f}_{x}$ equal $\frac{1}{2}$ only when the nodes are located in the center of their control volumes. Note that $f_{x}$ and $\tilde{f}_{x}$ are different geometric factors. Let us remind that the 
harmonic/arithmetic mean is the standard practice for the interpolated coefficient in the steady equation. When the unsteady factor is added we cannot apply the same practice because $\Delta M$ is an extensive variable for which the use of the geometric factor is not appropriate. Arguably, the coefficient can also be considered a partially extensive variable if the finite volume integration is not divided by the cell volume (as in this work), so even in this case the standard practice might not be fully appropriate. However, there are additional hypotheses involved in the derivation of an interpolation à la Rhie-Chow as arguable as this one so a thorough discussion on this issue may not be warranted and the preference for any interpolation has to be neccesarily connected to its accuracy and consistency.

We have employed two different interpolation modes. The first one is the standard approach for $A_{P \mid e}^{u}$ and the $\tilde{f}_{x}$-expression for $\Delta M_{e}$. This can be interpreted as an intensive practice for $A_{P \mid e}^{u}$ and a mass-consistent evaluation for $\Delta M_{e}$. The factor $\delta_{e}$ that appears in many of the formulae is calculated as

$$
\delta_{e}=\frac{\rho_{e} \Delta V_{e}}{\Delta t A_{P \mid e}^{u}}=\frac{\Delta M_{e}}{\Delta t A_{P \mid e}^{u}} \quad \widetilde{A}_{P \mid e}^{u}=\left(1+\delta_{e}\right) A_{P \mid e}^{u}
$$

The second approach originates from the study carried out with the inconsistent schemes that is described in a following section. We interpolate the coefficient per unit mass in this way

$$
\widetilde{A}_{P \mid e}^{u}=\left[\frac{A_{P \mid e}^{u}}{\rho_{e} \Delta V_{e}}+\frac{1}{\Delta t}\right] \rho_{e} \Delta V_{e}=\left[\overline{\left(\frac{A_{P \mid i}^{u}}{\Delta M_{i}}\right)^{e}}+\frac{1}{\Delta t}\right] \Delta M_{e}
$$

and

$$
A_{P \mid e}^{u}=\overline{\left(\frac{A_{P \mid i}^{u}}{\Delta M_{i}}\right)^{e}} \Delta M_{e}
$$

$\delta_{e}$ is calculated as $\delta_{e}=\left(\widetilde{A}_{P \mid e}^{u} / A_{P \mid e}^{u}\right)-1$ and $\Delta M_{e}$ is calculated as before. This second approach will be shown to satisfy the interpolation required to make some schemes consistent, i.e., free of erroneous dependencies. When discretizing we do not divide by the cell volume but if the equations are discretized in each finite volume and then divided by the volume itself the two approaches are equivalent for an incompressible case.

Finally, a word of caution is warranted: the coding must be self consistent. We encountered convergence problems if we did not follow a consistent practice and that made us spend some time in assessing the alternatives. Moreover, in some instances to be discussed it is imperative to use one specific interpolation as the others would generate unwanted dependencies. 


\section{The good practice for the interpolation of velocities}

The objective of this section is to show that there is a very simple criterium to ascertain if a given interpolation practice will unconditionally be time step independent. The criterium is stated as follows:

"If in the (steady state) limit when $u^{*}=u^{l}=u^{n}$ the expression for $u_{e}$ takes the same form as its corresponding steady equation, the scheme is time step independent"

This is a neccesary and sufficient condition for a scheme to be $\Delta t$ independent. Evidently if some underrelaxation is incorporated the form should be that of the nonrelaxed steady equation. Once read the criterium appears rather trivial but surprisingly it is not always satisfied by some of the schemes proposed in the literature. Some adaptation might be required and we will discuss ways of making $\Delta$ t-independent schemes that do not look as such at first sight. We will label schemes that satisfy the criterium with no required adaptation as 'unconditionally consistent'.

First we will show that Kadja et al's interpolation, Eq. 42, is correct in the sense that produces a unique steady state solution. We must note that the steady expression may also be dependent on the scheme. For instance, for this last scheme is

$$
A_{P \mid e}^{u} u_{e}^{*}={\overline{A_{P \mid i}^{u} u_{i}^{*}}}^{e}+\left[\overline{\left.\Delta V_{i} \frac{\partial p}{\partial x}\right|_{i} ^{e}}-\left.\Delta V_{e} \frac{\partial p}{\partial x}\right|_{e}\right]
$$

but for PICTURE should be

$$
u_{e}^{*}={\overline{u_{i}^{*}}}^{e}+\left[\overline{\left.\frac{\Delta V_{i}}{A_{P \mid i}^{u}} \frac{\partial p}{\partial x}\right|_{i} ^{e}}-\left.\frac{\Delta V_{e}}{A_{P \mid e}^{u}} \frac{\partial p}{\partial x}\right|_{e}\right]
$$

Let us remind that $A^{u}$ does not contain the temporal factor. Kadja et al's scheme is written as

$$
\begin{aligned}
& \widetilde{A}_{P \mid e}^{u} u_{e}^{*}={\widetilde{A_{P \mid i}^{u} u_{i}^{*}}}^{e}+\alpha_{u}\left[\left.\Delta V_{i} \frac{\partial p}{\partial x}\right|_{i} ^{l}{ }^{e}-\left.\Delta V_{e} \frac{\partial p}{\partial x}\right|_{e} ^{l}\right]+ \\
& +\left(1-\alpha_{u}\right)\left(\widetilde{A}_{P \mid e}^{u} u_{e}^{l}-{\widetilde{\widetilde{A}_{P \mid i}^{u} u_{i}^{l}}}^{e}\right)+ \\
& +\alpha_{u}\left(\frac{\rho_{e} \Delta V_{e}}{\Delta t} u_{e}^{n}-{\overline{\rho_{i} \Delta V_{i}}}^{\Delta t} u_{i}^{n}\right)
\end{aligned}
$$


When $u^{l}$ is equal to $u^{*}$ we can sum up the first terms on both sides and the third term on the RHS to obtain

$$
\begin{aligned}
\alpha_{u} \widetilde{A}_{P \mid e}^{u} u_{e}^{*} & =\alpha_{u}{\widetilde{A_{P \mid i}^{u} u_{i}^{*}}}^{e}+\alpha_{u}\left[{\overline{\left.\Delta V_{i} \frac{\partial p}{\partial x}\right|_{i} ^{l}}}^{e}-\left.\Delta V_{e} \frac{\partial p}{\partial x}\right|_{e} ^{l}\right]+ \\
& +\alpha_{u}\left(\frac{\rho_{e} \Delta V_{e}}{\Delta t} u_{e}^{n}-\frac{{\overline{\rho_{i} \Delta V_{i}}}_{\Delta t} u_{i}^{n}}{e}\right)
\end{aligned}
$$

$\alpha_{u}$ can be crossed out and as $u^{n}=u^{*}$ and $\widetilde{A}=A+\rho \Delta V / \Delta t$ the final expression is the same as Eq. 55, so the scheme is unconditionally consistent ${ }^{4}$. After a little algebra it can be seen that the new interpolation PICTURE presented in this paper also satisfies the criterium. However, if we rearrange Choi's scheme in the limit $u^{*}=u^{l}=u^{n}$ we end up with

$$
u_{e}^{*}=\overline{\frac{1+\delta_{e}}{1+\delta_{i}} u_{i}^{*}}+\overline{\left.\frac{1+\delta_{e}}{1+\delta_{i}} \frac{\Delta V_{i}}{A_{P \mid i}^{u}} \frac{\partial p}{\partial x}\right|_{i} ^{l}}-\left.\frac{\Delta V_{e}}{A_{P \mid e}^{u}} \frac{\partial p}{\partial x}\right|_{e} ^{l}
$$

and the factor $\left(1+\delta_{e}\right) /\left(1+\delta_{i}\right)$ makes the final solution to be time step dependent. The dependence is more evident for small time steps. Only in the limit $\Delta t \rightarrow \infty\left(\delta_{i}, \delta_{e} \rightarrow 0\right)$ the scheme works well, but in that case we are not dealing with a true transient situation. The scheme, as will be explained in the next section, is conditionally consistent, meaning that it is imperative to use a certain interpolator to make it consistent.

This insatisfactory behaviour can be traced back to the denominator of the interpolation equation

$$
\frac{\sum_{j \mid e} A_{j}^{u} u_{j}^{*}+S_{e}^{u}}{\widetilde{A}_{P \mid e}^{u}}=\overline{\left(\frac{\sum_{j \mid i} A_{j}^{u} u_{j}^{*}+S_{i}^{u}}{\widetilde{A}_{P \mid i}^{u}}\right)^{e}}
$$

and more precisely to its time dependency, something that was not present in the first scheme. In a previous paragraph it was mentioned that if we included the contribution from the previous iteration in the interpolated $H_{e}$ the resultant final solution would be relaxation-dependent. The cause and the consequence in a transient case go in parallel with that statement. If there is any non-multiplicative $\Delta$ t factor in the term to be interpolated the resultant scheme will be $\Delta \mathrm{t}$ dependent. The precise meaning of the adjective non-multiplicative will be clear in a moment. Let us advance that $\widetilde{A}$ contains a non-multiplicative time factor whereas $A$ does not, and $\sum_{i \mid e} A_{i}^{u} u_{i}^{*}+S_{e}^{u}$ does

$\overline{4}$ In the results section we will show that this is completely true as long the e-volume is calculated in a certain way 
not either. In the next section this assertion will be qualified because with a little extra work we can make any scheme free of unwanted $\Delta t$ dependence.

PICTURETWO gives when $u^{*}=u^{l}=u^{n}$

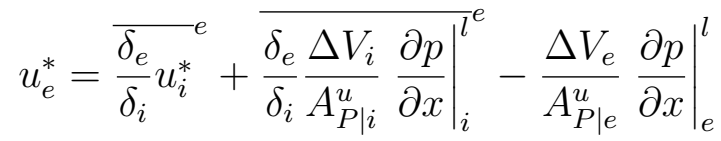

The factor $\delta_{e} / \delta_{i}$ makes the solution inconsistent with the steady expression. However this ratio does not contain any time factor because

$$
\frac{\delta_{e}}{\delta_{i}}=\frac{\rho_{e} \Delta V_{e}}{\rho_{i} \Delta V_{i}} \frac{A_{P \mid i}^{u}}{A_{P \mid e}^{u}}
$$

so the final solution will be independent of $\Delta \mathrm{t}$. One may have suspected a certain time step dependency on noticing a $\Delta t$ in the term interpolated in Eq. 45 but as a matter of fact this is not the source of the inconsistency. As long as $\Delta t$ appears as a uniform multiplicative factor in that term ${ }^{5}$, that is, a common factor that multiplies the whole term, the interpolation can be understood as being performed over what rests after removing $\Delta t$. The reason why PICTURETWO is inconsistent is that its solution in the steady limit does not correspond to the steady solution, unless $\rho_{i} \Delta V_{i}=\rho_{e} \Delta V_{e}$ in which case is identical to Kadja et al's scheme. We can thus assure that in a general case the PICTURETWO solution will be independent of $\Delta \mathrm{t}$ but dependent on the cell mass variation ${ }^{6}$ because Eq. 61 can always be written as

$$
A_{P \mid e}^{u} u_{e}^{*}=\overline{m r_{i} A_{P \mid i}^{u} u_{i}^{*}} e^{e}+\left[\left.m r_{i} \Delta V_{i} \frac{\partial p}{\partial x}\right|_{i} ^{l}-\left.\Delta V_{e} \frac{\partial p}{\partial x}\right|_{e} ^{l}\right]
$$

$m r_{i}$ being the mass ratio, $\left(\rho_{e} \Delta V_{e}\right) /\left(\rho_{i} \Delta V_{i}\right)$. Compare this with Eq. 55. Looking back to Eq. 45 one realizes that if the cell masses are equal the interpolated term is proportional to $\sum_{i \mid e} A_{i}^{u} u_{i}^{*}+S_{e}^{u} \Delta V_{e}$ so the equivalence to Kadja et al's approach is obvious. Incidentally, this exemplifies the generality of the proposed criterium by which one can detect dependencies other than those on the time step not obvious in the first place.

\footnotetext{
$\overline{5}$ As is $\alpha_{u}$ when dealing with the relaxed steady equation

6 This is directly related to the expansion/contraction ratio of the grid in an incompressible case.
} 


\section{A unified formulation for all schemes. How to make consistent an otherwise inconsistent scheme.}

In this section a unification of all schemes will be described leading to interpolation criteria that will make both Choi's and PICTURETWO schemes free of erroneous dependencies. Let us start with the general equation for $u_{e}^{*}$ divided through by a generic function $\phi_{e}$. This function is specific of every scheme.

$$
\begin{aligned}
\frac{\widetilde{A}_{P \mid e}^{u}}{\alpha_{u} \phi_{e}} u_{e}^{*} & =\frac{\sum_{j \mid e} A_{j}^{u} u_{j}^{*}+S_{e}^{u} \Delta V_{e}}{\phi_{e}}-\left.\frac{\Delta V_{e}}{\phi_{e}} \frac{\partial p}{\partial x}\right|_{e} ^{l}+ \\
& +\frac{1-\alpha_{u}}{\alpha_{u}} \frac{\widetilde{A}_{P \mid e}^{u} u_{e}^{l}}{\phi_{e}}+\frac{\rho_{e} \Delta V_{e}}{\Delta t \phi_{e}} u_{e}^{n}
\end{aligned}
$$

Similar equations can be written for $u_{P}^{*}$ and $u_{E}^{*}$. The arithmetic interpolation is realized over the first term on the RHS and the generic $u_{e}^{*}$ expression to be implemented is

$$
\begin{aligned}
& \frac{\widetilde{A}_{P \mid e}^{u}}{\phi_{e}} u_{e}^{*}={\widehat{\widetilde{A}_{P \mid i}^{u}}}_{\phi_{i}} u_{i}^{*}+\alpha_{u}\left[{\widehat{\left.\frac{\Delta V_{i}}{\phi_{i}} \frac{\partial p}{\partial x}\right|_{i} ^{l}}}_{i}^{e}-\left.\frac{\Delta V_{e}}{\phi_{e}} \frac{\partial p}{\partial x}\right|_{e} ^{l}\right]+ \\
& +\left(1-\alpha_{u}\right)\left(\frac{\widetilde{A}_{P \mid e}^{u}}{\phi_{e}} u_{e}^{l}-{\widehat{{\widetilde{A_{P \mid i}^{u}}}^{l}}}_{\phi_{i}^{l}}^{l}\right)+\alpha_{u}\left(\frac{\rho_{e} \Delta V_{e}}{\Delta t \phi_{e}} u_{e}^{n}-{\overline{\frac{\rho_{i} \Delta V_{i}}{\Delta t \phi_{i}}}}_{i}^{e}\right)
\end{aligned}
$$

The four schemes are obtained if

$$
\begin{array}{llll}
\phi_{e, i} & =A_{P \mid e, i}^{u} & \text { PICTURE } & \phi_{e, i}=1 \quad \text { Kadja } \\
\phi_{e, i}=\widetilde{A}_{P \mid e, i}^{u} & \text { Choi } & \phi_{e, i}=\frac{\rho_{e, i} \Delta V_{e, i}}{\Delta t} & \text { PICTURETWO }
\end{array}
$$

Note that we do not preclude any average, that is, the widehat-overline can represent a geometric average or otherwise. As a matter of fact, to make the problematic schemes consistent we will have to resort to employing weighting factors other than geometric. We will discuss different alternatives to obtain a consistent steady solution and in order to do so we have to derive generic solutions when $u_{e, i}^{*}=u_{e, i}^{l}=u_{e, i}^{n}$. Operating with the previous equation it is easy to show that these are

$$
\frac{A_{P \mid e}^{u}}{\phi_{e}} u_{e}^{*}={\widehat{\frac{A_{P \mid i}^{u}}{\phi_{i}} u_{i}^{*}}}_{e}+\left[\left.\overline{\frac{\Delta V_{i} \frac{\partial p}{\phi_{i}} \frac{{ }^{l}}{\partial x}}{e}}\right|_{i} ^{e}-\left.\frac{\Delta V_{e}}{\phi_{e}} \frac{\partial p}{\partial x}\right|_{e} ^{l}\right]
$$


We must remember that this final solution has to be equivalent to Eq. 55 or Eq. 56. It is inmediate to check that if one uses a geometric average both PICTURE and Kajda schemes meet this requirement but Choi and PICTURETWO do not. The question we posit is: Is there any other average to be used in these schemes that leads to consistency?. The answer is yes and we can find it by simple comparison with the consistent formulations. Let us take PICTURE for instance. Its steady state expression is

$$
u_{e}^{*}={\overline{u_{i}^{*}}}^{e}+\left[\overline{\left.\frac{\Delta V_{i}}{A_{P \mid i}^{u}} \frac{\partial p}{\partial x}\right|_{i} ^{l}}-\left.\frac{\Delta V_{e}}{A_{P \mid e}^{u}} \frac{\partial p}{\partial x}\right|_{e} ^{l}\right]
$$

Comparing this equation with Eqn. (67) it is realized that the average proposed should be one that satisfies

$$
{\widehat{\frac{A_{P \mid i}^{u}}{\phi_{i}} u_{i}^{*}}} \propto \overline{u_{i}^{*}}
$$

because in that case the first term in the RHS of (67) will be the same as in PICTURE. The following average for a generic function $\zeta$ meets the requirement just mentioned

$$
\begin{aligned}
& \left.\widehat{{\overline{\zeta_{i}}}^{e}}={\overline{\phi_{i}}}_{A_{P \mid i}^{u}}^{e} / \overline{\left(\frac{\phi_{i}}{A_{P \mid i}^{u}}\right)}\right)^{e} \Rightarrow \widehat{{\overline{\zeta_{i}^{e}}}^{e}}=\frac{{\overline{\left(1+\delta_{i}\right) \zeta_{i}}}^{e}}{{\overline{\left(1+\delta_{i}\right)^{e}}}^{e}} \quad \text { Choi } \\
& \widehat{{\overline{\zeta_{i}}}^{e}}=\frac{{\overline{\delta_{i} \zeta_{i}}}^{e}}{{\overline{\delta_{i}}}^{e}} \quad \text { PICTURETWO }
\end{aligned}
$$

the denominator is included to preserve the property that the average of a constant is the same constant. This average has as weighting factors for Choi and PICTURETWO

$$
\widehat{f}_{x}=\frac{\left(1+\delta_{P}\right) f_{x}}{\left(1+\delta_{P}\right) f_{x}+\left(1+\delta_{E}\right)\left(1-f_{x}\right)} \quad, \quad \frac{\delta_{P} f_{x}}{\delta_{P} f_{x}+\delta_{E}\left(1-f_{x}\right)}
$$

With this average all terms in the inconsistent schemes expressions are also identical to those of PICTURE. Let us take for instance the pressure term in Choi's expression, Eq. 39. The overlines represent the proposed non-geometric interpolation, then

$$
\left.\alpha_{u} \Delta t \overline{\frac{\delta_{i}}{\rho_{i}\left(1+\delta_{i}\right)}} \frac{\partial p}{\partial x}\right|_{i} ^{l}=\alpha_{u} \Delta t \overline{\left.\frac{\delta_{i}\left(1+\delta_{i}\right)}{\rho_{i}\left(1+\delta_{i}\right)} \frac{\partial p}{\partial x}\right|_{i} ^{l^{e}}}=\left.\alpha_{u} \Delta t \overline{\frac{\delta_{i}}{\rho_{i}} \frac{\partial p}{\partial x}}\right|_{i} ^{l^{e}}
$$


which is the PICTURE term. To be consistent with all e-located terms in the general $u_{e}^{*}$ expression of PICTURE the factors at $e$ have to be calculated as

$$
\frac{\phi_{e}}{A_{P \mid e}^{u}}={\overline{\left(\frac{\phi_{i}}{A_{P \mid i}^{u}}\right)^{e}}}^{e}
$$

For both Choi and PICTURETWO this reduces to $\delta_{e}=\bar{\delta}_{i}{ }^{e}$. There is no other restriction. Observe that we can still use an arithmetic average for $A_{P \mid e}^{u}$ (if needed) but in that case for both schemes to be consistent $\widetilde{A}_{P \mid e}^{u}$ should be calculated as

$$
\widetilde{A}_{P \mid e}^{u}=A_{P \mid e}^{u}\left(1+\delta_{e}\right)={\overline{A_{P \mid i}^{u}}}^{e}\left(1+{\overline{\delta_{i}}}^{e}\right)
$$

and not with the traditional geometric average

$$
\widetilde{A}_{P \mid e}^{u}={\widetilde{\widetilde{A}_{P \mid i}^{u}}}^{e}
$$

The work presented in this section was prompted by the fact that in some test cases with the inconsistent schemes we obtained solutions closer to the exact one when the second interpolation practice presented in a previous section was employed. When using the first interpolation mode the discrepancy was manifest, the results being quite far from the exact ones. This did not happen by chance and it took us some time to understand the whole scenario and to be able to make PICTURETWO and Choi's schemes consistent. In fact, according to Eqn (73) the consistent PICTURE-equivalent interpolation for both is

$$
\frac{\Delta M_{e}}{A_{P \mid e}^{u}}=\overline{\left(\frac{\Delta M_{i}}{A_{P \mid i}^{u}}\right)^{e}}
$$

So, we are obliged to average the coefficient per unit mass for the schemes to be consistent ${ }^{7}$. That is why this interpolation resulted in a much better solution.

For the sake of completeness let us present the equivalent averages and restrictions for the schemes to be consistent with Kadja et al's scheme.

$\overline{7}$ Strictly speaking, we used the arithmetic mean for the inverse which is the same as the harmonic mean in Eqn. 76. 


$$
\begin{aligned}
& \widehat{{\overline{\zeta_{i}}}^{e}}=\frac{{\overline{\phi_{i} \zeta_{i}}}^{e}}{{\overline{\phi_{i}}}^{e}} \Rightarrow \widehat{\bar{\zeta}_{i}^{e}}=\frac{{\widetilde{A_{P \mid i}^{u} \zeta_{i}}}^{e}}{{\widetilde{A_{P \mid i}^{u}}}^{e}} \quad \text { Choi } \\
& \widehat{{\overline{\zeta_{i}}}^{e}}=\frac{{\overline{\Delta M_{i} \zeta_{i}}}^{e}}{{\overline{\Delta M_{i}}}^{e}} \quad \text { PICTURETWO }
\end{aligned}
$$

with the restriction

$$
\begin{aligned}
\phi_{e}=\bar{\phi}_{i}^{e} \Rightarrow \widetilde{A}_{P \mid e}^{u} & ={\overline{\widetilde{A}_{P \mid i}^{u}}}^{e} \quad \text { Choi } \\
\Delta M_{e} & ={\overline{\Delta M_{i}}}^{e} \quad \text { PICTURETWO }
\end{aligned}
$$

We must stress that these restrictions only apply to the inconsistent schemes. In principle, and in terms of consistency, any average is allowed for the elocated factors in PICTURE and almost anyone in Kadja et al's scheme.

If SIMPLER scheme is to be employed the application of consistent interpolations is crucial for the computations to have physical sense. One has to be specially careful as the expression for the $u_{e}^{*}$ velocity that serves to derive the discrete pressure equation contains factors that are interpolated differently within each scheme. To be more specific, let us write the $u_{e}^{*}$ equation that is common for all schemes

$$
u_{e}^{*}=\alpha_{u} \frac{\phi_{e}}{\widetilde{A}_{P \mid e}^{u}} \overline{H_{i}^{*}}{ }^{e}-\left.\alpha_{u} \frac{\Delta t}{\rho_{e}} \frac{\delta_{e}}{1+\delta_{e}} \frac{\partial p}{\partial x}\right|_{e} ^{l}+\left(1-\alpha_{u}\right) u_{e}^{l}+\alpha_{u} \frac{\delta_{e}}{1+\delta_{e}} u_{e}^{n}
$$

with

$$
{\overline{H_{i}^{*}}}^{e}={\overline{\left(\frac{\sum_{j \mid i} A_{j}^{u} u_{j}^{*}+S_{i}^{u} \Delta V_{i}}{\phi_{i}}\right)}}^{e}
$$

Except the first term on the RHS the rest are the same for all schemes because they do not depend on the particular $\phi$ function employed. We have to interpolate the factors at $e$ that appear in this equation, that is, $\phi_{e} / \widetilde{A}_{P \mid e}^{u}$ and $\delta_{e}$. The first factor is 1 in Choi's case and $\delta_{e} /\left(1+\delta_{e}\right)$ in PICTURETWO so we have to look for consistent interpolations for $\delta_{e}$. For a PICTURE-equivalent solution there is no problem because it can be consistently calculated using $\delta_{e}=\bar{\delta}_{i}{ }^{e}$. For a solution Kadja-equivalent we will distinguish between Choi and PICTURETWO because apparently either restriction is different, although it will allow a unified formulation. For Choi

$$
\widetilde{A}_{P \mid e}^{u}={\overline{\widetilde{A}_{P \mid i}^{u}}}^{e} \Rightarrow A_{P \mid e}^{u}+\frac{\rho_{e} \Delta V_{e}}{\Delta t}=\overline{A_{P \mid i}^{u}+\frac{\rho_{i} \Delta V_{i}}{}}{ }^{e}={\overline{A_{P \mid i}^{u}}}^{e}+\frac{{\overline{\rho_{i} \Delta V_{i}}}^{e}}{\Delta t}
$$


So, it seems logical to identify

$$
A_{P \mid e}^{u}={\overline{A_{P \mid i}^{u}}}^{e} \quad ; \quad \frac{\rho_{e} \Delta V_{e}}{\Delta t}=\frac{{\overline{\rho_{i} \Delta V_{i}}}^{e}}{\Delta t}
$$

and to calculate $\delta_{e}$ as

$$
\delta_{e}=\frac{\overline{\rho_{i} \Delta V_{i}^{e}}}{\Delta t \bar{A}_{P \mid i}^{u} e}
$$

For PICTURETWO the restriction is

$$
\rho_{e} \Delta V_{e}=\overline{\rho_{i} \Delta V_{i}^{e}} \Rightarrow \widetilde{A}_{P \mid e}^{u}-A_{P \mid e}^{u}={\overline{\widetilde{A}_{P \mid i}^{u}-A_{P \mid i}^{u}}}^{e}={\overline{\widetilde{A}_{P \mid i}^{u}}}^{e}-{\overline{A_{P \mid i}^{u}}}^{e}
$$

so, as before, it seems logical to identify

$$
\widetilde{A}_{P \mid e}^{u}={\widetilde{A_{P \mid i}^{u}}}^{e} \quad ; \quad A_{P \mid e}^{u}={\overline{A_{P \mid i}^{u}}}^{e}
$$

and the unified formulation for both is

$$
\widetilde{A}_{P \mid e}^{u}={\overline{\widetilde{A}_{P \mid i}^{u}}}^{e} \quad ; \quad A_{P \mid e}^{u}={\overline{A_{P \mid i}^{u}}}^{e} \quad ; \quad \rho_{e} \Delta V_{e}=\overline{\rho_{i} \Delta V_{i}^{e}} \quad ; \delta_{e}=\frac{\overline{\rho_{i} \Delta V_{i}^{e}}}{\Delta t \overline{A_{P \mid i}^{u}}(86)}
$$

Incidentally, in the interpolations $\rho_{e} \Delta V_{e}$ may sometimes lose its mass-conserving expression in favour of being consistent. It is better to think of $\rho_{e} \Delta V_{e}$ as a variable with a required interpolation for consistency (as is $\widetilde{A}_{P \mid e}^{u}$ ) than to assign a real identity to such 'fictitious' variable.

It is convenient at this point to summarize the ideas discussed in this section. With traditional geometric averages the two inconsistent schemes will always produce solutions dependent either on the time step or the spatial step. For consistency, i.e., the final solution independent of these steps, all widehat averages for generic terms $\zeta$ have to be averaged with Eqn. (70) or (77). Also, all e-factors contained in the $u_{e}^{*}$ general expression have to be calculated based on the restrictions given in Eqn. (73) or (78) depending on the specific function $\phi$. It is straightforward to see that all these special averaging procedures make both Choi and PICTURETWO equivalent to PICTURE or Kadja et al's scheme because the time dependent expressions for $u_{e}^{*}$ are equal, as well as the steady state expressions. Although the consistent formulations of the inconsistent schemes are identical term by term with either PICTURE or Kadja we will continue calling these schemes with the original name, acknowledging the fact that we have employed the same coding for all formulations. The 
only part we have changed is the function that evaluates the weighting factors which are no longer exclusively geometrical, as Eq. 71 indicates.

In this section a relatively easy way of transforming inconsistent schemes into consistent ones has been presented. Of course, there is no point in using inconsistent schemes if later one seeks equivalent consistent expressions by choosing appropriate weighting factors so this thorough discussion is only relevant to those who have already implemented inconsistent schemes and want to obtain correct results. There is an easy way out of the inconsistency: the interpolations and the weighting factors need to be changed. To this author's knowledge nobody has previously addressed the issue of the interpolation operators and their fundamental effect in the solution, at least with the inconsistent schemes.

All schemes will be compared in the next section in steady examples. Our intention is to utilize examples to support our claims and these are related to the good/bad use of pseudotime marching schemes with relaxation in problems moving towards steady state. Of course a scheme that produces steady solutions that are time step dependent cannot be recommended for unsteady problems so all comments in this paper are also relevant in unsteady situations. The discussion on the various alternatives of the transient $u_{e}^{*}$ is important in itself because, especially with Choi's scheme, there has been some controversy over the last years, with conflicting claims. In some of the revised papers no explicit mention of the interpolation employed is made ${ }^{8}$ so it is difficult to know whether the conflict is a consequence of the various interpolations. In any event, those that specify the interpolation using Choi's scheme employ a traditional geometric weighting for all averages. The solution in this case is not consistent as the required interpolations and weighting factors are not adequate.

\section{Results}

All schemes will be applied to the solution of a simplified one dimensional flow, which was employed formerly by Date [32] in a report on PV coupling in colocated grids. This flow, although simple, contains all the essential features of the pressure velocity coupling and can highlight the pros and cons of the approaches described. The continuity and Navier-Stokes equation to be discretized are

$$
\frac{d}{d x} \rho u=\dot{m}
$$

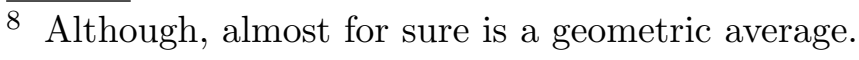




$$
\frac{d}{d x} \rho u^{2}=-\frac{d p}{d x}+\dot{m} u+\mu \frac{d^{2} u}{d x^{2}}+S_{m}
$$

These equations govern the motion of an incompressible 1D flow with mass and momentum injection. $\rho$ may be considered as a line density $(\mathrm{kgr} / \mathrm{m})$ and $\dot{m}$ represents the mass source $(\mathrm{kgr} / \mathrm{m} / \mathrm{s}$ injected to or extracted from the 1D domain) which may depend on $x$ and/or $t$. In the Navier-Stokes equation the momentum source is $\dot{m} u$ as each $\mathrm{kgr} / \mathrm{m} / \mathrm{s}$ of fluid injected/extracted at one point has to bring in/take away $\dot{m} u$ momentum units per meter and per second, otherwise the variable $u$ would have two different values at the same point. $S_{m}$ represents additional momentum sources due to gravity forces and other body forces in non-inertial reference frames, if any. By adjusting appropriately the source terms a wealth of different (continuous and discontinuous) analytic solutions may be obtained that will allow us to compare the different unsteady interpolations. All physical properties have been taken as constants of value one and the inlet conditions are $u_{o}=1$ and $p_{o}=0$.

We will show cases where the inconsistency is revealed and where the changes brought about by consistent interpolations are more noticeable. The three cases tested correspond to different mass and momentum source functions. There is no special reason behind the use of this concrete blend of sources but the fact that they have been employed, among others, as computational exercices in a CFD course taught by the author. Arguably, these cases are neither computationally demanding (good) nor numerically demanding (not that good), except perhaps the first case because of the discontinuity in the pressure field. However, the main purpose of this section is just to support the theoretical findings which form the core of the paper and these examples provide clear answers to the questions raised in this work. To show that a scheme is $\Delta \mathrm{t}$ or $\Delta \mathrm{V}$ dependent can perfectly be realized in rather simple onedimensional flows as the ones picked up. If there are any doubts cast on whether a consistent scheme in $1 \mathrm{D}$ will remain so in $2 \mathrm{D}$ or $3 \mathrm{D}$ problems we must point out that the cell face velocities in a colocated grid are always calculated averaging nodal values situated only along one coordinate, that perpendicular to the face. The cell face velocity is always calculated as if the problem was 1D. Thus from the point of view of PWIM interpolation one could say that $3 \mathrm{D}$ problems are $1+1+1 \mathrm{D}$.

The first test contains a constant mass and momentum source in the interval $(0.3,0.7)$ of a unity domain. The second one has only a linear momentum source in the same interval and the third one displays a linear mass and momentum sources from $\mathrm{x}=0.3$ until the end of the domain at $\mathrm{x}=1$. The exact solutions for the three cases tested are given in the Appendix. The computational results are always corresponding to a fully converged solution. If nothing is specified that means a residual below $10^{-14}$ for the mass imbalance and below $10^{-12}$ for the velocity equation, both in the energy norm. They are calculated as follows 


$$
\begin{aligned}
\text { massres } & =\sqrt{\sum_{P}\left|\left(\rho u_{e \mid P}-\rho u_{o \mid P}\right)-\dot{m} \Delta x\right|^{2}} \\
\text { velres } & =\sqrt{\sum_{P}\left|\frac{A_{P \mid P}^{u} u_{P}-\sum_{j \mid P} A_{j}^{u} u_{j}-S_{P}^{u}-G F^{u}\left(P_{w \mid P}-P_{e \mid P}\right)}{A_{P \mid P}^{u} u_{P}}\right|^{2}}
\end{aligned}
$$

In $S_{P}^{u}$ the contribution of the previous iteration and the preceding time step is not considered. In all figures the exact solution is shown as a solid line. Values at nodes and faces will be portrayed.

In Fig. A.1 the pressure distribution in the first case for several approaches is depicted. This case was one where the time dependency of Choi's scheme was evident. The grid points are distributed with the idea of combining uniform regions with others having a relatively rapid variation of the contraction/expansion (c/e) ratio. We found that in regions of rapid variation of the $\mathrm{c} / \mathrm{e}$ ratio the inconsistencies were more noticeable. There are 45 volumes of uniform size $\Delta x=0.01,5$ volumes with a contraction ratio of 0.8 (the first one with $\Delta x=0.01$ ), 6 volumes with an expansion ratio ${ }^{9}$ of 1.2 (the first one with $\Delta x=0.0033)$ and again a constant zone with $\Delta x=0.01$. Only the region of nonuniform spacing is presented. As we mentioned previously the inconsistency for Choi's scheme is more revealing as the time step is reduced. With $\Delta t=5 \cdot 10^{-4}$ the oscillations are pretty noticeable but they have increased dramatically for $\Delta t=10^{-4}$. In the figure, the solution of the modified Choi's approach, consistent with PICTURE and identical to it, is also shown. The oscillations are absent in this case and the solution lies very near the exact solution given by the solid line.

To understand why the effect of the inconsistency is more noticeable when $\Delta t$ is of order $10^{-4}$ or below, Fig A.2 shows the evolution of $\left(1+\delta_{e}\right) /\left(1+\delta_{i}\right), i=$ $E, P$, for four different $\Delta t: 10^{+5}, 10^{-4}, 10^{-5}$ and $10^{-10}, \delta_{e}$ being calculated as $\bar{\delta}_{i}{ }^{e}$. There are two factors associated to the same interface and for a given $\Delta t$ they are drawn in the figure as two lines with the same symbol. For a strict equivalence to PICTURE these factors should be 1 and they have in fact this value when the time step is $10^{+5}$, giving in that case a very smooth solution. As can be seen diminishing $\Delta t$ separates them more and more from unity. There is a direct link between this separation and the quality of the result: the more distance exists the more amplified the oscillations appear as these nonunity factors become dominant in the averaging process, vitiating the final solution. In the limit $\Delta t \rightarrow 0$ the factors are $\delta_{e} / \delta_{i}$. Let us remember that these are the ratios appearing in the inconsistent PICTURETWO. So, as a conclusion in this case, the oscillations produced by Choi's approach are of smaller or equal amplitude than those produced by PICTURETWO for all time steps.

$\overline{9}$ These ratios are in the limit of the recommended values for not loosing accuracy in the discretization. 
PICTURETWO results, shown in Fig A.3, corroborate this assertion as large amplitude oscillations are present. The complete unity domain is drawn to see the overall behaviour. The two solutions for different $\Delta t$ are the same, as expected, and the solution consistent with PICTURE is exactly the same as that of Choi's approach consistent with PICTURE.

In a true transient case it can be shown that $\delta$ is related to the inverse of the local CFL number (convective plus diffusive). In the case $\Delta t=5 \cdot 10^{-4}$, both $\delta$ and CFL are of order unity in most of the domain, the former decreasing significantly in the nonuniform part. There are two combined causes for the appearance of oscillations in Choi's scheme: small $\Delta t$ and significant variation of $\Delta V / A_{P}^{u}$. The combination of both makes $\delta$ become significant against unity with considerable changes in some regions and this causes the factors to move away from unity. To highlight the neccesity of both acting at the same time let us mention that we have obtained with Choi's inconsistent formulation a perfectly smooth solution for any $\Delta t$ (any CFL) in a uniform grid where $\Delta V / A_{P}^{u}$ hardly changes. The oscillations may appear if $\delta$ changes quickly in a significant manner, provided $\delta$ is not negligible compared to one. The cause may be a rapid variation of c/e ratio as in this case but it may well be quite another reason in a different problem. The oscillations will appear for CFL number roughly of order one, yet we must make clear that these oscillations have nothing to do with the explicitness of the time integration as the usual CFL condition has.

In Fig. A.4 the velocity is shown for the same case. The solution for Choi's inconsistent approach is almost indistinguishable from that of PICTURE, fact that highlights a systematic finding in this work: In all cases studied the inconsistency is always more reflected in the pressure than in the velocity, where is hardly noticeable sometimes. This statement is not intended to be general but it is important as Choi [11] and Yu et al. [17] [18] compared only velocities and not pressure. Finding tiny variations in the former does not neccesarily mean that the scheme is time step independent. Both variables have to be checked for time step independency. In Shen et al. [29] the lines where the dependency can be spotted are the isobars.

There are some situations where Kadja et al's approach shows oscillations although it is a consistent scheme. This case is one of them and it has to do with the intial assumption made in the scheme derivation. Let us remind that PICTURE assumes that $\left(\sum_{i \mid P} A_{i}^{u} u_{i}^{*}+S_{P}^{u}\right) / A_{P}^{u}$ is linear whereas Kadja et al's approach makes the same assumption for $\sum_{i \mid P} A_{i}^{u} u_{i}^{*}+S_{P}^{u}$. The oscillations can be directly related to the linearity of this term (or the lack thereof). In Fig. A.5 the solution with both schemes is presented with an inset for details and in Fig A.6 the term supposed to be linear is depicted. Over most part of the domain both solutions are indistinguishable but in the nonuniform region Kadja et al's result is much worse. It is obvious that Kadja et al's approach incorrectly 
assumes that $\sum_{i \mid P} A_{i}^{u} u_{i}^{*}+S_{P}^{u}$ is linear and this false assumption produces the oscillations in the nonlinear region. The local maxima and minima in the solution are related to intervals where the second derivative of the addend is large in relative terms. On the other hand, PICTURE assumption is correct over the whole domain resulting in a smooth solution. It was mentioned in a previous section that there are consistent schemes that can produce oscillations, now it is understood that these are partially linked ${ }^{10}$ to the adequacy of the linearity assumption implicit in the derivation of the cell face velocity.

The second case presented in Fig A.7 also reveals the inconsistency of Choi's scheme. This is a case for which the solution is very smooth but the grid adopted has rapid variations in the cell size, $\Delta V$, in order to produce large changes in $\delta$. The grid is contracting with a contraction ratio of 0.8 up to $x=0.5$ and then expanding with an expansion ratio of 1.2 until $x=1$. The scheme is run with different $\Delta t: 10^{-4}, 10^{-3}$ and $10^{+5}$. The solutions with the first two $\Delta t$ are a complete nonsense, neither the tendency nor the values are reproduced. The solution for $\Delta t=10^{+5}$, in which $\delta$ is very small compared to one, lies on top of the exact solution as does the consistent alternative for any $\Delta t$ (not shown). A companion figure is Fig A.8 where PICTURETWO results with $\Delta t=10^{-4}, 10^{+5}$ are presented along with the solution obtained with PICTURETWO consistent with PICTURE. Again, the solutions of the inconsistent scheme, identical for both $\Delta t$, is far off the exact one. Near 0.5 there are a great many points lying outside the $y$ axis range as the actual pressure range of the solution goes from -10000 to 15000. Apparently the effect of the ratio change at 0.5 gives rise to huge oscillations near this point and vitiates the solution all over the domain. Let us remember that PICTURETWO is unduly dependent on the finite volume size variation. To check the theoretical finding of independency of PICTURETWO from $\Delta t, 10$ runs with $\Delta t$ ranging from $10^{-5}$ to $10^{2}$ in one order of magnitude intervals, plus $10^{5}$ and $10^{25}$, were carried out obtaining (bad) solutions differing only from the 12 th decimal place onwards.

The velocity in this second case with Choi's scheme is presented in Fig. A.9. As there is no mass source the velocity solution at the cell interfaces has to be constantly equal to the inlet velocity, otherwise the scheme would not conserve mass. It is well known that, unlike the face velocity, the cell velocity does not conserve mass in a colocated arrangement because the continuity equation is not applied to control volumes having the cell velocities on their faces. In fact these are calculated with their discretized transport equation. Due to the wrong coupling between faces and nodes the results show a velocity field quite spiky. This is a clear example of a very simple flow in which both pressure and velocity behave erratically owing to the inconsistencies of the transient

\footnotetext{
${ }^{10}$ We say 'partially linked' because there are other required interpolations in the $u_{e}^{*}$ expression that can affect the amplitude of the oscillations, if any.
} 
formulation.

In the course of this work we came across several unexpected oscillatory solutions of the consistent Kadja scheme. One of them was the first test case, discussed and explained before, another is next one. This third computational case will allow us to compare the different alternatives one has for the evaluation of some of the terms at the cell interface of the consistent schemes. We will see that no matter how we evaluate $\delta_{e}$ for PICTURE the steady state result is the same, but it is not so for Kadja et al's scheme. For the latter it is important to caution against an arbitrary evaluation of the factors associated to the cell face because if they are not computed properly the solution, otherwise smooth, can present some oscillations.

To start with the discussion let us write again PICTURE expression for the face velocity

$$
\begin{aligned}
\left(1+\delta_{e}\right) u_{e}^{*} & =\overline{\left(1+\delta_{i}\right) u_{i}^{*}}+\alpha_{u} \Delta t\left[\overline{\left.\frac{\delta_{i}}{\rho_{i}} \frac{\partial p}{\partial x}\right|_{i} ^{l}}-\left.\frac{\delta_{e}}{\rho_{e}} \frac{\partial p}{\partial x}\right|_{e} ^{l}\right]+ \\
& +\left(1-\alpha_{u}\right)\left[\left(1+\delta_{e}\right) u_{e}^{l}-\overline{\left(1+\delta_{i}\right) u_{i}^{l}}\right]+\alpha_{u}\left[\delta_{e} u_{e}^{n}-{\overline{\delta_{i} u_{i}^{n}}}^{e}\right]
\end{aligned}
$$

Rearranging this equation when $u_{e}^{*}=u_{e}^{l}=u_{e}^{n}$ it can be seen that the final steady state expression is

$$
u_{e}^{*}={\overline{u_{i}^{*}}}^{e}+\left[\overline{\left.\frac{\Delta V_{i}}{A_{P \mid i}^{u}} \frac{\partial p}{\partial x}\right|_{i} ^{e}}-\left.\frac{\Delta V_{e}}{A_{P \mid e}^{u}} \frac{\partial p}{\partial x}\right|_{e}\right]
$$

independently of the way $\delta_{e}$ is actually evaluated. Kadja et al's expression is

$$
\begin{aligned}
A_{P \mid e}^{u}\left(1+\delta_{e}\right) u_{e}^{*} & \left.=\overline{A_{P \mid i}^{u}\left(1+\delta_{i}\right) u_{i}^{*}}+\alpha_{u} \overline{\left[\left.\Delta V_{i} \frac{\partial p}{\partial x}\right|_{i} ^{l}\right.}-\left.\Delta V_{e} \frac{\partial p}{\partial x}\right|_{e} ^{l}\right]+ \\
& +\left(1-\alpha_{u}\right)\left(A_{P \mid e}^{u}\left(1+\delta_{e}\right) u_{e}^{l}-\overline{A_{P \mid i}^{u}\left(1+\delta_{i}\right) u_{i}^{l}}{ }^{e}\right)+ \\
& +\alpha_{u}\left(A_{P \mid e}^{u} \delta_{e} u_{e}^{n}-\overline{A_{P \mid i}^{u} \delta_{i} u_{i}^{n}}\right)
\end{aligned}
$$

At the end of the iterations within a time step, $u_{e}^{*}=u_{e}^{l}$, the expression is

$$
\begin{aligned}
A_{P \mid e}^{u}\left(1+\delta_{e}\right) u_{e}^{*} & =\overline{A_{P \mid i}^{u}\left(1+\delta_{i}\right) u_{i}^{*}}+\left[\overline{\left.\Delta V_{i} \frac{\partial p}{\partial x}\right|_{i} ^{l}}-\left.\Delta V_{e} \frac{\partial p}{\partial x}\right|_{e} ^{l}\right] \\
& +\left(A_{P \mid e}^{u} \delta_{e} u_{e}^{n}-{\overline{A_{P \mid i}^{u} \delta_{i} u_{i}^{n}}}^{e}\right)
\end{aligned}
$$


The correct expression at steady state, $u_{e}^{*}=u_{e}^{n}$, is

$$
A_{P \mid e}^{u} u_{e}^{*}=\overline{A_{P \mid i}^{u} u_{i}^{*}}+\left[\overline{\left.\Delta V_{i} \frac{\partial p}{\partial x}\right|_{i} ^{l}}-\left.\Delta V_{e} \frac{\partial p}{\partial x}\right|_{e} ^{l}\right]
$$

So we have to look for interpolations that satisfy at steady state

$$
A_{P \mid e}^{u}\left(1+\delta_{e}\right) u_{e}^{*}-A_{P \mid e}^{u} \delta_{e} u_{e}^{n}=A_{P \mid e}^{u} u_{e}
$$

that is the same as

$$
A_{P \mid e}^{u}\left(1+\delta_{e}\right)-A_{P \mid e}^{u} \delta_{e}=\widetilde{A}_{P \mid e}^{u}-\frac{\rho_{e} \Delta V_{e}}{\Delta t}=A_{P \mid e}^{u}
$$

As odd as it seems not all interpolations satisfy this trivial equality. For instance if we used

$$
\widetilde{A}_{P \mid e}^{u}={\overline{\widetilde{A}_{P \mid i}^{u}}}^{e}={\overline{A_{P \mid i}^{u}}}^{e}+\frac{\overline{\rho_{i} \Delta V_{i}^{e}}}{\Delta t} \quad \text { and } \quad \rho_{e} \Delta V_{e}=\widetilde{f}_{x}\left(\Delta M_{P}+\Delta M_{E}\right)
$$

this interpolation would result in the following steady state expression

$$
\left({\overline{A_{P \mid i}^{u}}}^{e}+\frac{\overline{\rho_{i} \Delta V_{i}^{e}}}{\Delta t}-\frac{\rho_{e} \Delta V_{e}}{\Delta t}\right) u_{e}^{*}={\overline{A_{P \mid i}^{u} u_{i}^{*}}}^{e}+\left[\overline{\left.\Delta V_{i} \frac{\partial p}{\partial x}\right|_{i} ^{l}}-\left.\Delta V_{e} \frac{\partial p}{\partial x}\right|_{e} ^{l}\right]
$$

The problem arises if we use a nonuniform grid and $\Delta V_{e}$ is calculated in a massconsistent way as $\rho_{e} \Delta V_{e}=\tilde{f}_{x}\left(\Delta M_{P}+\Delta M_{E}\right)$. In that case the second and third terms in the LHS would not compensate each other and its difference would give rise to a nonunique steady state solution dependent on the time step. Yet for a transient flow in a colocated grid this is the usual approach with Kadjalike schemes. For instance, Yu et al [17] and some other researchers interpolate $\widetilde{A}_{P \mid e}^{u}$ and use a mass-consistent evaluation of $\Delta V_{e}$, this may sometimes produce oscillations and time-step dependent solutions. To corroborate this analysis let us choose an extremely simple flow: a constant velocity field with a constant momentum source of value one hundred between 0.3 and 0.7 . The momentum source makes the pressure gradient to be constant in that interval. We will use a grid similar to that of the first test case. The results of pressure are portrayed in Fig. A.10 and those of velocity in Fig. A.11. For the oscillations to appear the difference between $\overline{\rho_{i} \Delta V_{i}^{e}}$ and $\rho_{e} \Delta V_{e}$, both divided by $\Delta t$, has to be comparable to ${\overline{A_{P \mid i}^{u}}}^{e}$, this requires a relatively small time step and a strong nonuniform grid. Results are shown for $\Delta t=5 \cdot 10^{-7}$ and $10^{-6}$. For this latter time step the interpolation that makes the difference equal to zero is also 
shown. Two aspects stand out: the solution dependency on the time step and the disappearance of the oscillations when a mass-inconsistent interpolation is employed. As commented previously we have sometimes to abandon the idea of $\Delta V_{e}$ being a 'real' volume size.

Because of the possibility of Kadja et al's scheme to produce oscillations it is not recommended for general use. We recognize that this last inconsistency encountered is very subtle, we came across it by sheer coincidence on using very small time steps. In any event, there is an alternative free of inconsistencies: PICTURE.

\section{Conclusions}

In this paper a thorough review of different proposals presented in the literature that deal with the (pseudo)unsteady Navier-Stokes equations in a colocated grid has been accomplished. By doing so, a general criterium to ascertain if a given scheme will be free of erroneous dependencies has been put forward and under this criterium the inconsistencies of former schemes have been highlighted. A new unconditionally consistent scheme has been derived and compared with others of the same class. It can be considered as the logical extension to transient problems of the Rhie-Chow procedure. This consistent scheme always provides time independent solutions, unlike other consistent schemes in the literature that sometimes result in oscillatory and time dependent solutions. Finally, a new manner of turning inconsistent schemes into consistent ones by using special interpolation practices has been explained.

We hope to have clarified a subject that to our understanding remained a bit obscure: the extension to (pseudo)time dependent problems of the procedures peculiar to a colocated grid. In particular, the consistent evaluation of the (pseudo)time dependent cell face velocity without introducing unreal dependencies.

\section{A Exact solutions of the computational tests}

In the first computational case we have the (constant) mass and momentum sources active in the interval $\left(x_{1}, x_{2}\right)$ in a domain of unit length. The boundary conditions for velocity and pressure on the left boundary are $u=u_{o}$ and $p=p_{o}$. In the interval between 0 and $x_{1}$ there is no source present so the solution is $u=$ const. $=u_{o}, p=$ const. $=p_{o}$. At $x=x_{1}$ there is a discontinuity in the $u$ derivative from 0 to $\dot{m} / \rho$. This can be represented mathematically with the Heaviside step function whose derivative is the delta function. That means 
that at $x=x_{1}$ the viscous term becomes $\frac{\dot{m} \mu}{\rho} \delta\left(x-x_{1}\right)$. This delta function has to be compensated by the pressure gradient and consequently the pressure must also be a step function of amplitude $\dot{m} \mu / \rho$, that is, $p\left(x_{1}^{+}\right)=p_{o}+\dot{m} \mu / \rho$. In the interval where both sources are present the solution is

$$
\begin{aligned}
& u(x)=u_{o}+\frac{\dot{m}}{\rho}\left(x-x_{1}\right) \\
& p(x)=p_{o}+\frac{\dot{m} \mu}{\rho}+\left(S_{m}-\dot{m} u_{o}\right)\left(x-x_{1}\right)-\frac{\dot{m}^{2}}{2 \rho}\left(x-x_{1}\right)^{2}
\end{aligned}
$$

At $x=x_{2}$ there is another delta function coming from the viscous term so there is a new (negative) step in the pressure of the same amplitude. From $x=x_{2}$ onwards the solution is $p=p\left(x_{2}^{+}\right)$and $u=u\left(x_{2}^{+}\right)$.

Summing up, the solution of the first computational case is

$$
\begin{aligned}
0 \leqslant x \leqslant x_{1}^{-} \quad u(x)=u_{o} \quad p(x)=p_{o} \\
x_{1}^{+} \leqslant x \leqslant x_{2}^{-} \quad u(x)=u_{o}+\frac{\dot{m}}{\rho}\left(x-x_{1}\right) \\
p(x)=p_{o}+\frac{\dot{m} \mu}{\rho}+\left(S_{m}-\dot{m} u_{o}\right)\left(x-x_{1}\right)-\frac{\dot{m}^{2}}{2 \rho}\left(x-x_{1}\right)^{2} \\
x_{2}^{+} \leqslant x \leqslant 1 . \quad u(x)=u_{o}+\frac{\dot{m}}{\rho}\left(x_{2}-x_{1}\right) \\
p(x)=p_{o}+\left(S_{m}-\dot{m} u_{o}\right)\left(x_{2}-x_{1}\right)-\frac{\dot{m}^{2}}{2 \rho}\left(x_{2}-x_{1}\right)^{2}
\end{aligned}
$$

The particular values of the variables employed in the first computational case are

$$
\dot{m}=S_{m}=u_{o}=\rho=\mu=1 \quad ; \quad p_{o}=0 \quad ; \quad x_{1}=0.3, x_{2}=0.7
$$

.The second case involves only a linear momentum source, $S_{m}=S_{m o}(x-$ $\left.x_{1}\right) /\left(x_{2}-x_{1}\right)$, active between $x_{1}$ and $x_{2}$. This source distribution produces a quadratic dependence for the pressure in that interval, this variable being continuous over the domain because there is no mass source. The velocity and pressure solutions are

$$
\begin{array}{rl}
0 \leqslant x \leqslant 1 & u(x)=u_{o} \\
0 \leqslant x \leqslant x_{1} & p(x)=p_{o} \\
x_{1} \leqslant x \leqslant x_{2} & p(x)=p_{o}+\frac{S_{m o}}{2} \frac{\left(x-x_{1}\right)^{2}}{x_{2}-x_{1}} \\
x_{2} \leqslant x & p(x)=p_{o}+\frac{S_{m o}}{2}\left(x_{2}-x_{1}\right)
\end{array}
$$


The particular values of the variables employed in the second computational case are

$$
S_{m o}=u_{o}=\rho=\mu=1 \quad ; \quad \dot{m}=p_{o}=0 \quad ; \quad x_{1}=0.3, x_{2}=0.7
$$

\section{References}

[1] C. M. Rhie, W. L. Chow, Numerical Study of the Turbulent Flow Past an Airfoil with Trailing Edge Separation, AIAA J. vol 21, issue 11, pp 1525-1532, 1983

[2] S. Majumdar, Role of Underrelaxation in Momentum Interpolation for Calculations of Flows with Nonstaggered Grids, Numer. Heat Transfer vol 13, pp 125-132, 1988

[3] T. F. Miller, F. W. Schmidt, Use of a Pressure-Weighted Interpolation Method for the Solution of the Incompressible Navier-Stokes Equations on a Nonstaggered Grid System, Numer. Heat Transfer vol 14, pp 213-233, 1988

[4] G. D. Thiart, Finite Difference Scheme for the Numerical Solution of Fluid Flow and Heat Transfer Problems on Nonstaggered Grids, Numer. Heat Transfer, part B, vol 17, pp 43-62, 1990

[5] G. W. Wang, J. G. Wei, W. Q. Tao, An Improved Numerical Algorithm for Solution of Convective Heat Transfer Problems on Nonstaggered Grid System, Heat and Mass Transfer, vol 33, pp 273-280, 1998

[6] H. Aksoy, C. S. Chen, Numerical Solution of Navier-Stokes Equations with Nonstaggered Grids using Finite Analytic Method, Numer. Heat Transfer, Part B, vol 21, pp 287-306, 1992

[7] A. Miettinen, A Study of the Pressure Correction Approach in the Colocated Grid Arrangement, Lic. Thesis, Helsinki University of Technology, 1997

[8] A. W. Date, Complete Pressure Correction Algorithm for Solution of Incompressible Navier-Stokes Equations on a Nonstaggered Grid, Numer. Heat Transfer, Part B, vol 29, pp 441-458, 1996

[9] A. W. Date, Solution of Navier-Stokes Equations on Nonstaggered Grid, Int. J. Heat Mass Transfer vol 36, pp 1913-1922, 1993

[10] H. Xu, C. Zhang, Study of the Effect of the Non-orthogonality for Non-staggered Grids-The Theory, Int. J. Numer. Meth. Fluids, vol 28, issue 9, pp 1265-1280, 1998

[11] S. K. Choi, Note on the Use of Momentum Interpolation Method for Unsteady Flows, Numer. Heat Transfer, Part A, vol 36, pp 545-550, 1999

[12] Y. G. Lai, R. M. C. So, A. J. Prezkwas, Turbulent Transonic Flow Simulation Using a Pressure-Based Method, Int. J. Engng Sci., vol 33, No. 4, pp 469-483, 1995 
[13] A. Cubero, N. Fueyo, A Compact Momentum Interpolation Procedure for Unsteady Flows and Relaxation, Numerical Heat Transfer, Part B, vol 52, pp 507-529, 2007

[14] M. Kadja, J. S. Anagnostopoulos, G. C. Bergeles, Study of Wind Flow and Pollutant Dispersion by Newly Developed Precision-Improving Methods, Int. Comm. Heat Mass Transfer, vol 23, issue 8, pp 1065-1076, 1996

[15] M. Kadja, J. S. Anagnostopoulos, G. C. Bergeles, Implementation of Newly Developed Algorithms in the Simulation of Atmospheric Turbulent Transports, Computers \& Fluids, vol 26, issue 5, pp 489-504, 1997

[16] H. S. Udaykumar, H.-Ch. Kan, W. Shyy, R. Tran-Son-Tay, Multiphase Dynamics in Arbitrary Geometries on Fixed Cartesian Grids, J. of Comp. Phys., vol 137, pp 366-405, 1997

[17] B. Yu, Y. Kawaguchi, W.-Q. Tao, H. Ozoe, Checkerboard Pressure Predictions Due to the Underrelaxation Factor and Time Step Size for a Nonstaggered Grid with Momentum Interpolation Method, Numer. Heat Transfer, part B, vol 41, issue 1, pp 85-94, 2002.

[18] B. Yu, W.-Q. Tao, J.-J Wei, Discussion on Momentum Interpolation Method for Colocated Grids of Incompressible Flow, Numer. Heat Transfer, Part B, vol 42, pp 141-166, 2002

[19] I. E. Barton, R. Kirby, Finite Difference Scheme for the Solution of Fluid Flow Problems on Non-staggered Grids, Int. J. Numer. Meth. Fluids, vol 33, pp 939959,2000

[20] P. Johansson, L. Davidson, Modified Collocated SIMPLEC algorithm Applied to Buoyancy-affected Turbulent Flow using a Multigrid Solution Procedure, Numer. Heat Transfer, Part B, vol 28, pp 39-57, 1995

[21] M. Bohm, K. Weschler, M. Schafer, A Parallel Moving Grid Multigrid Method for Flow Simulation in Rotor-Stator Configurations, Int. J. Numer. Meth. Engng, vol 42, pp 175-189, 1998

[22] J. H. Ferziger, M. Peric, Computational Methods for Fluid Dynamics, 2nd edition, Springer Verlag, 1997

[23] M. Darwish, I. Sraj, F. Moukalled, A Coupled Incompressible Flow Solver on Structured Grids, Numer. Heat Transfer, Part B, vol 52, pp 353-371, 2007

[24] H. Nilsson, L. Davidson, CALC-PVM: A Parallel SIMPLEC Multiblock Solver for Turbulent Flow in Complex Domains. Chalmers University of Technology, Internal report Nr 98/12, 1998

[25] C.Y. Gu, Computation of Flows with Large Body Forces, in C. Taylor, J. H. Chin, G. M. Homsy (eds.), Numerical Methods in Laminar and Turbulent Flow, Pineridge Press, vol 7, part 2, pp 1568-1578, 1991

[26] S.-K. Choi, S.-O Kim, Ch.-H. Kim, H.-K. Choi, Use of the Momentum Interpolation Method for Flows with a Large Body Force, Numer. Heat Transfer, part B, vol 43, pp 267-287, 2003 
[27] M. M. Rahman, A. Miettinen, T. Siikonen, Modified SIMPLE formulation on a Collocated Grid with an Assessment of the Simplified QUICK Scheme, Numer. Heat Transfer, part B, vol 30, pp 291-314, 1996

[28] J. Mencinger, I. Z̆un, On the Finite Volume Discretization of Discontinuous Body Force Field on Collocated Grid: Application to VOF Method, J. of Comp. Phys., vol 221, pp 524-538, 2007

[29] W. Z. Shen, J. A. Michelsen, J. N. Sorensen, Improved Rhie-Chow Interpolation for Unsteady Flow Computations, AIAA J., vol 39, issue 12, pp 2406-2409, 2001

[30] W. Z. Shen, J. A. Michelsen, N. N. Sorensen, J. N. Sorensen, An Improved SIMPLEC Method on Collocated Grids for Steady and Unsteady Flow Computations, Numer. Heat Transfer, part B, vol 43, pp 221-239, 2003

[31] M. H. Kobayashi, J. C. F. Pereira, Numerical Comparison of Momentum Interpolation Methods and Pressure-Velocity Algorithms Using Non-Staggered Grids, Comm. in Appl. Num. Meth., vol 7, pp 173-186, 1991

[32] A.W. Date, On interpolation of cell-face velocities in the solution of N-S Equations using Nonstaggered Grids. Report SFB 210/T/79. University of Karlsruhe, 1991 


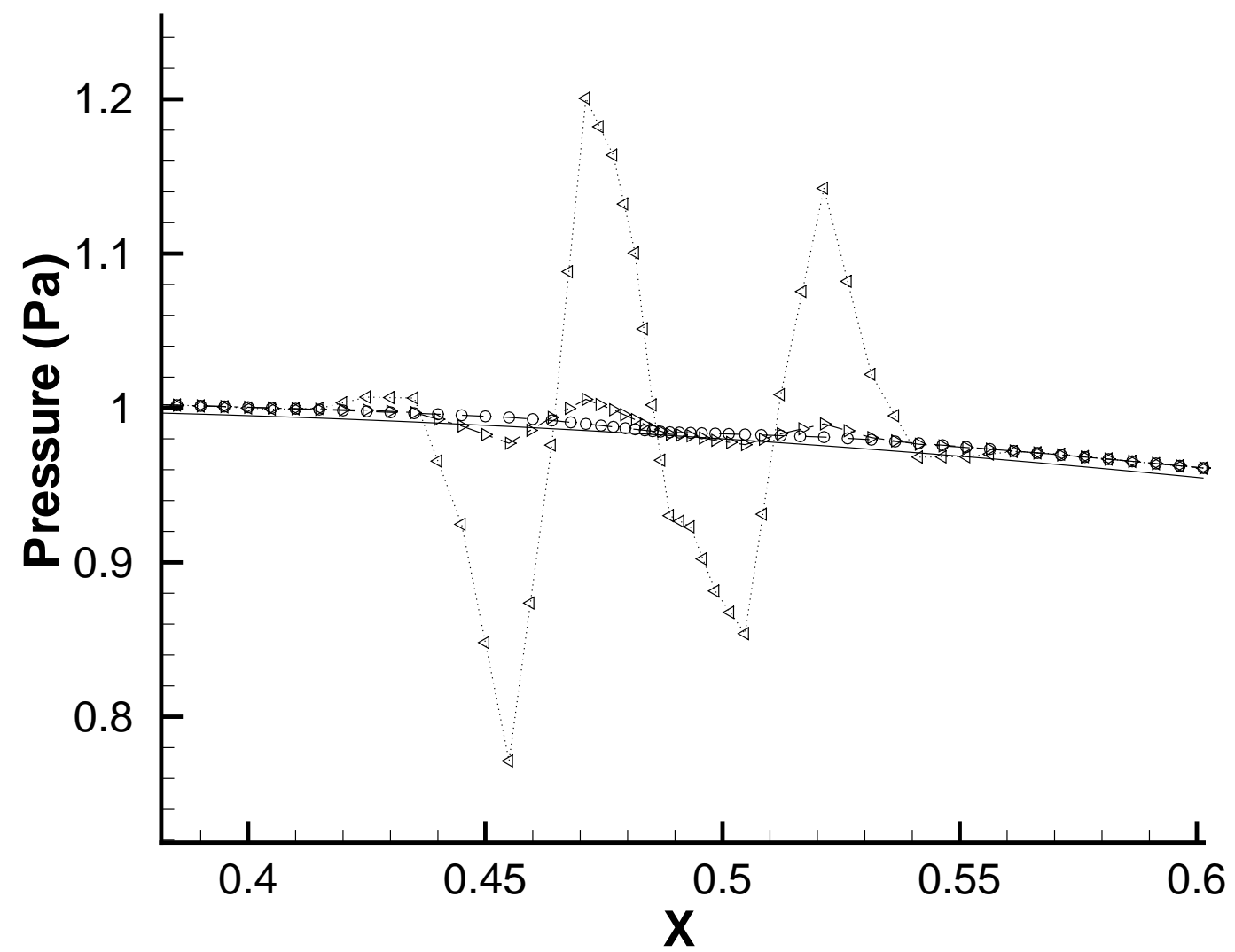

Fig. A.1. Pressure distribution for the first case with Choi's scheme. $\triangleleft$ Choi's inconsistent approach $\Delta t=10^{-4}$; $\triangleright$ Choi's inconsistent approach $\Delta t=5.10^{-4}$; O Choi's approach consistent with PICTURE. 


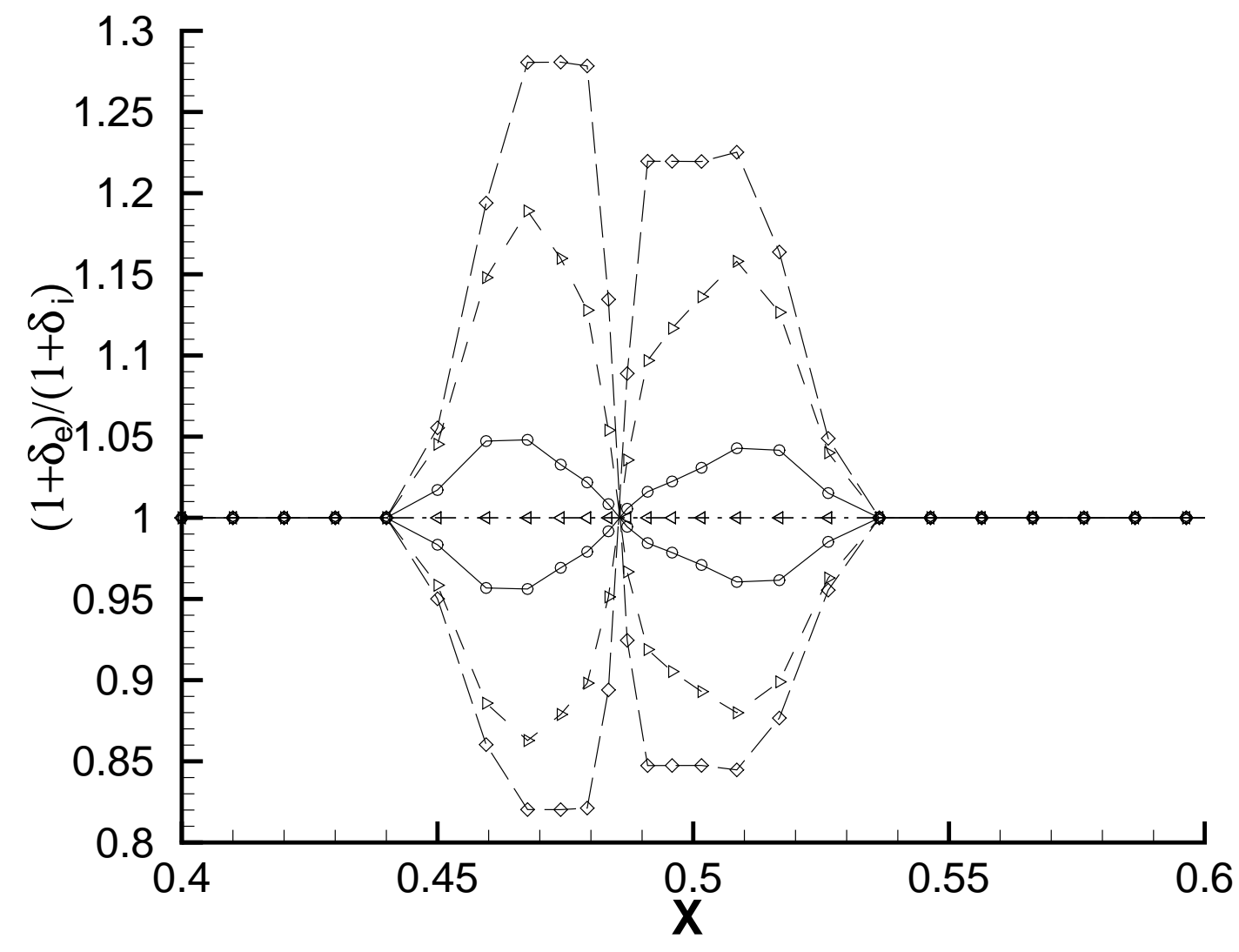

Fig. A.2. Factors dependence on $\Delta t . \triangleleft \Delta t=10^{+5} ; \circ \Delta t=10^{-4} ; \triangleright \Delta t=10^{-5}$; $\diamond \Delta t=10^{-10}$ 


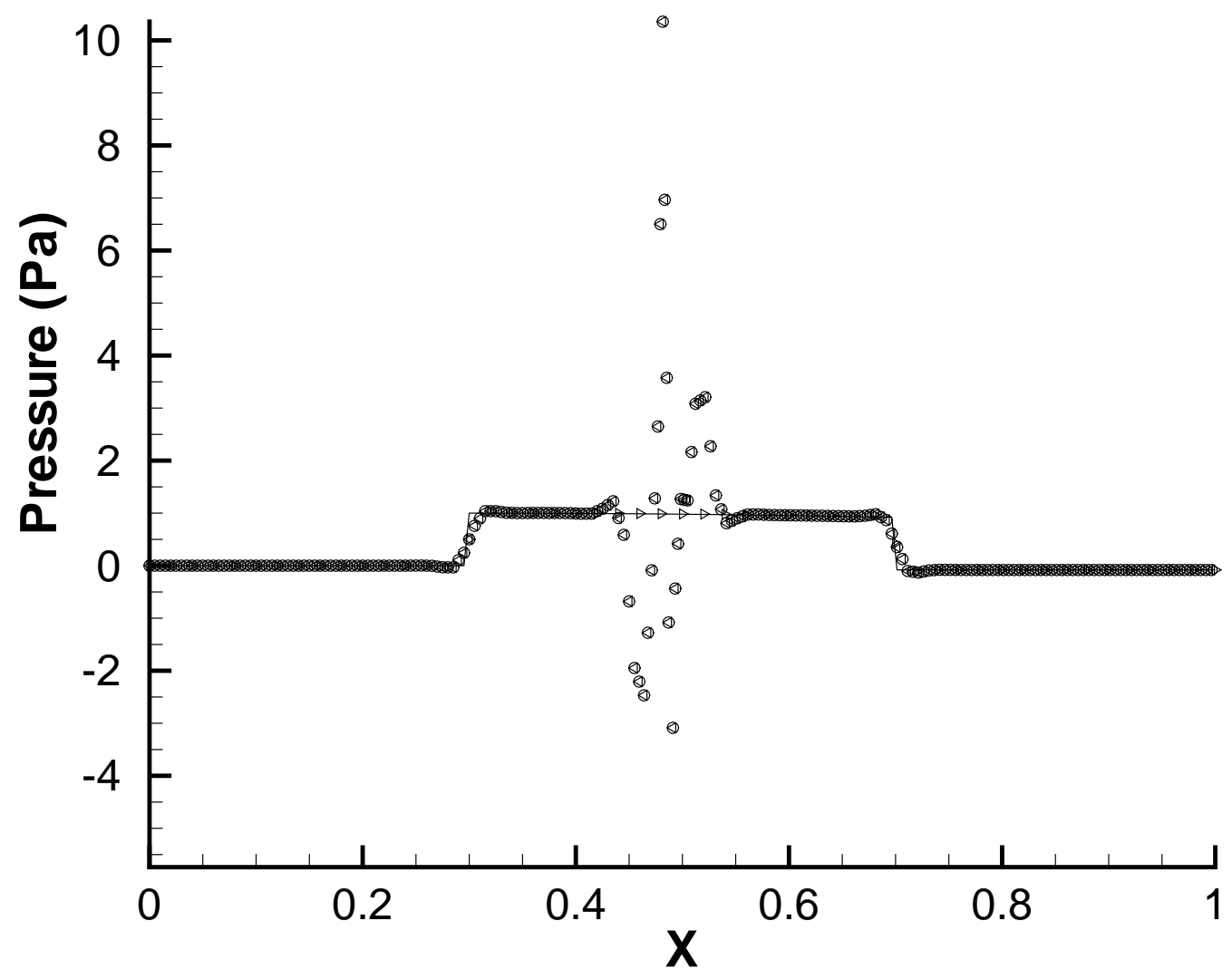

Fig. A.3. Pressure distribution with PICTURETWO. $\triangleright$ PICTURETWO consistent with PICTURE; O PICTURETWO inconsistent, $\Delta t=10^{+5} ; \triangleleft$ PICTURETWO inconsistent, $\Delta t=10^{-5}$. 


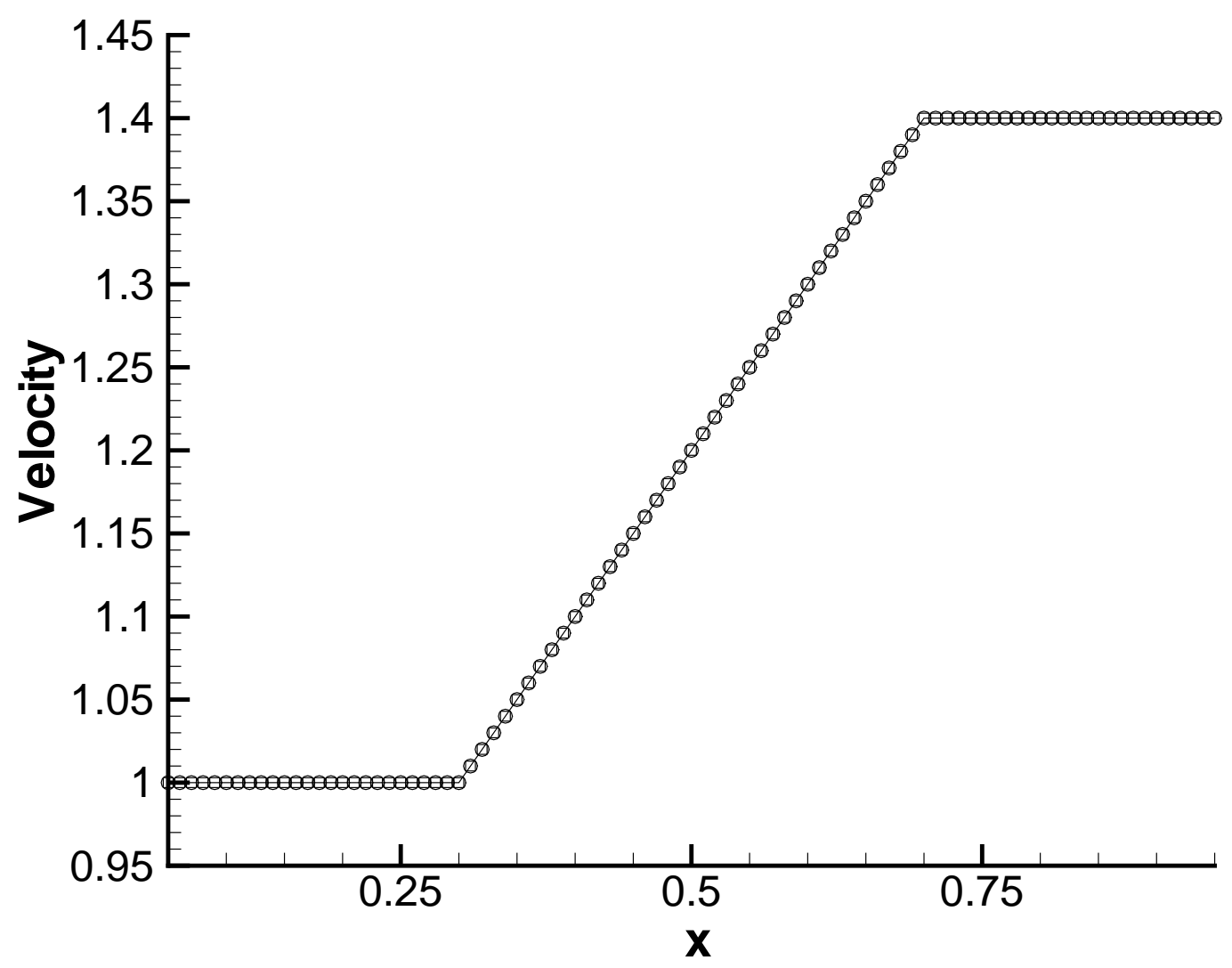

Fig. A.4. Velocity distribution in the first case. O PICTURE; $\square$ Choi inconsistent, $\Delta t=10^{-5}$. 


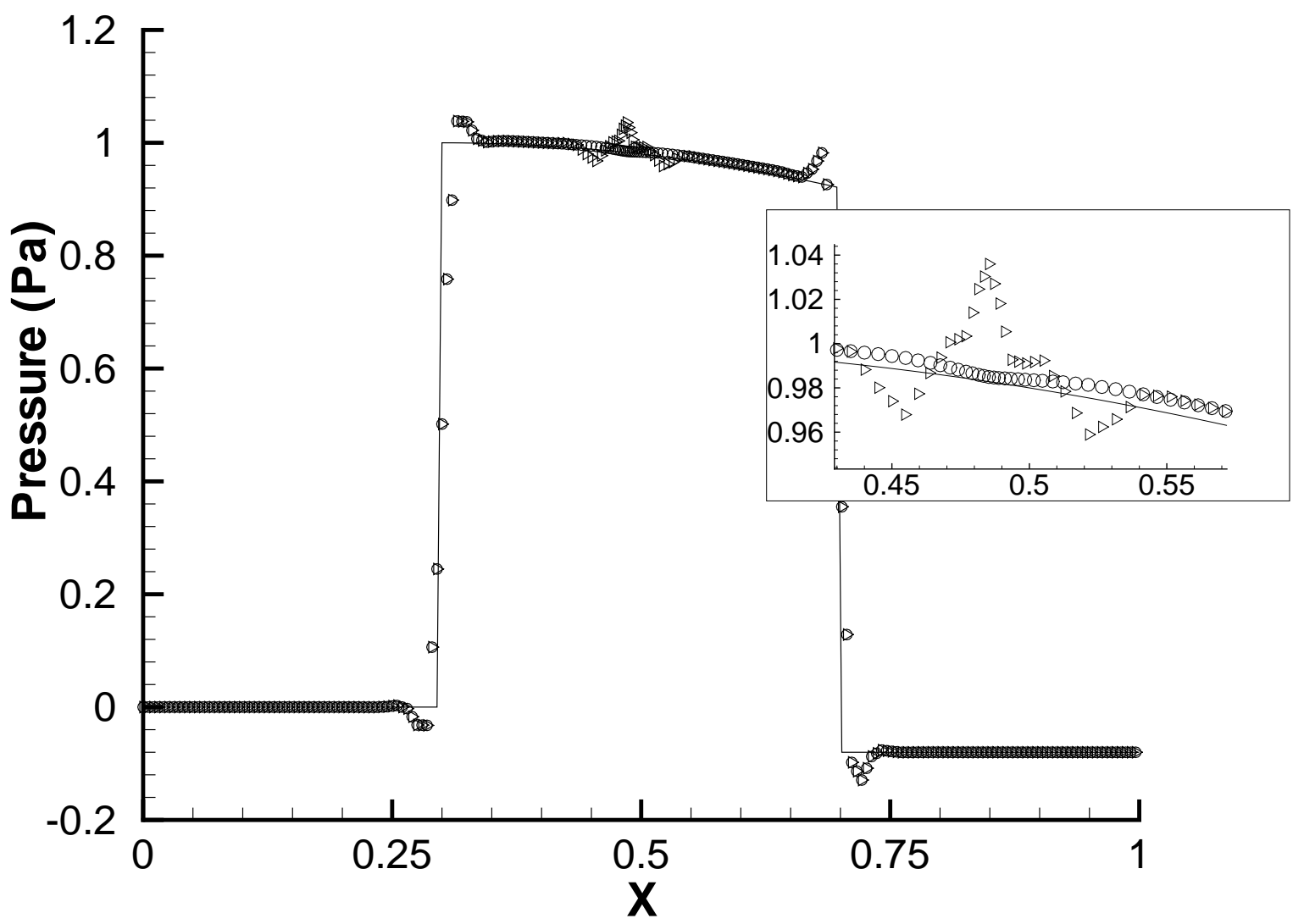

Fig. A.5. Pressure distribution for the first case with the two consistent schemes. O PICTURE; $\triangleright$ Kadja. 


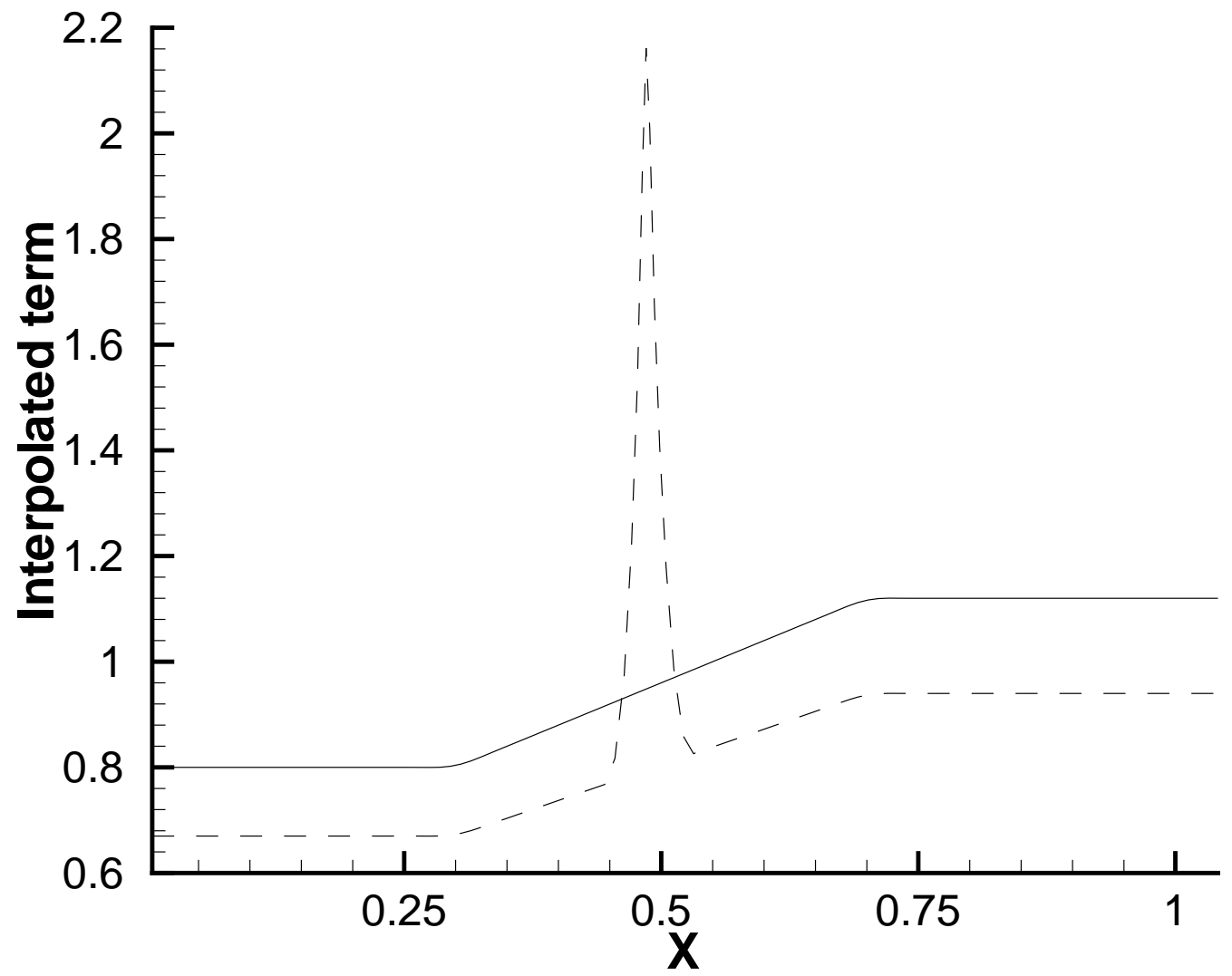

Fig. A.6. Interpolated term in the first case. Solid line $\left(\sum_{j \mid P} A_{j}^{u} u_{j}^{*}+S_{P}^{u}\right) / A_{P \mid P}^{u}$. Dashed line $\sum_{j \mid P} A_{j}^{u} u_{j}^{*}+S_{P}^{u}$. 


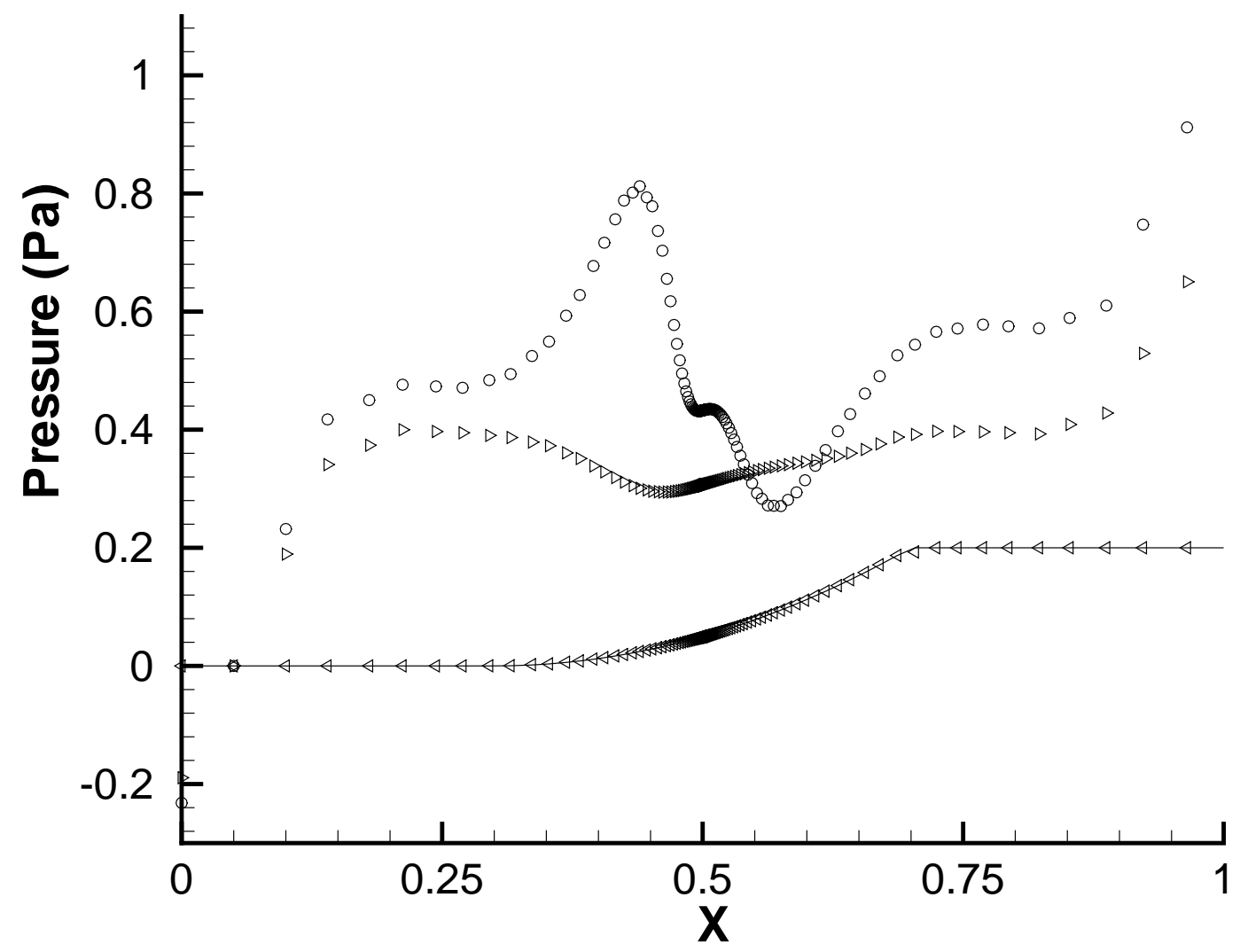

Fig. A.7. Pressure for the second case. $\bigcirc$ Choi inconsistent $\Delta t=10^{-4}$; $\triangleright$ Choi inconsistent $\Delta t=10^{-3} ; \triangleleft$ Choi inconsistent $\Delta t=10^{+5}$; 


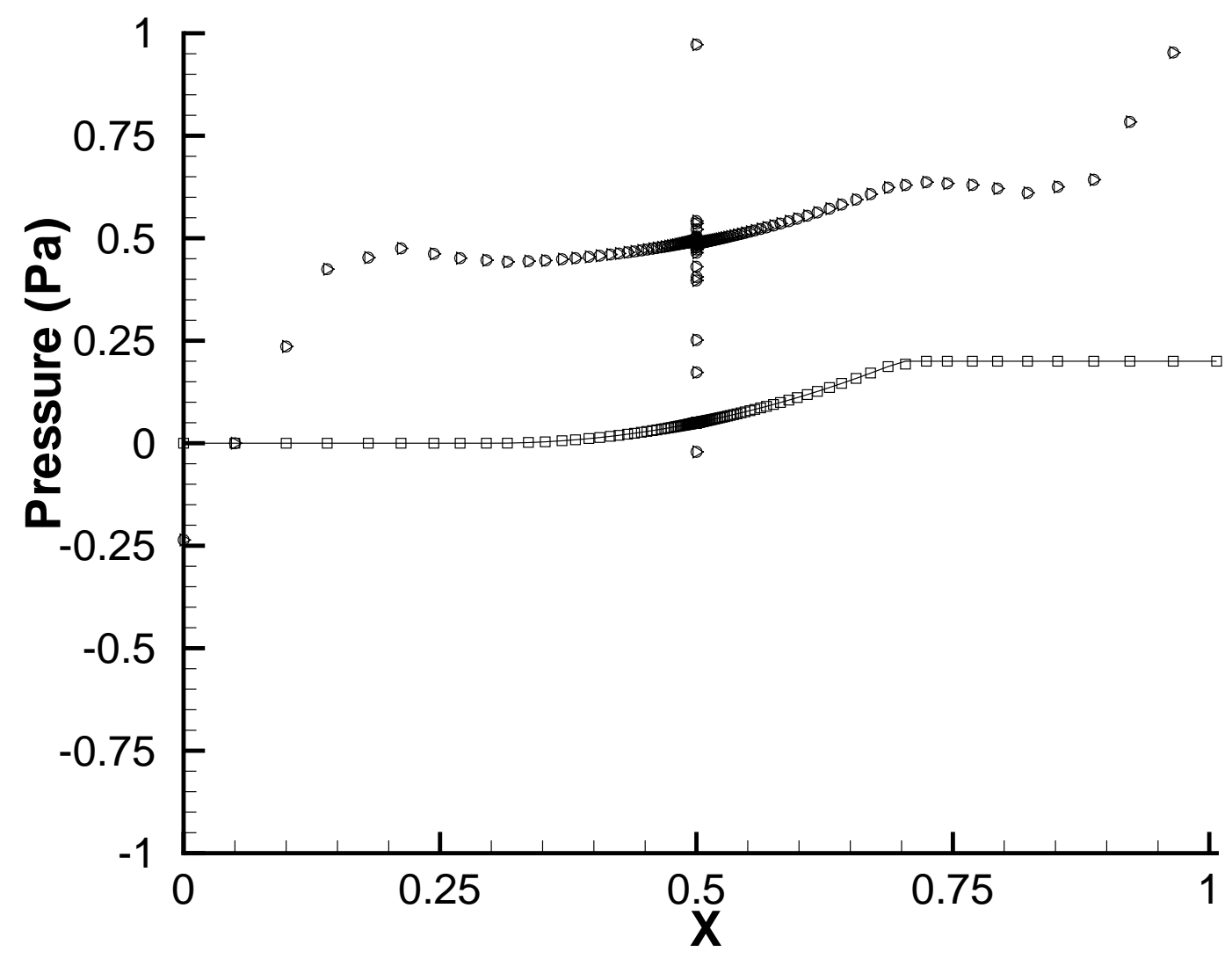

Fig. A.8. Pressure for the second case. O PICTURETWO inconsistent $\Delta t=10^{-4} ; \triangleright$ PICTURETWO inconsistent $\Delta t=10^{+5} ; \square$ PICTURETWO consistent $\Delta t=10^{+5}$; 


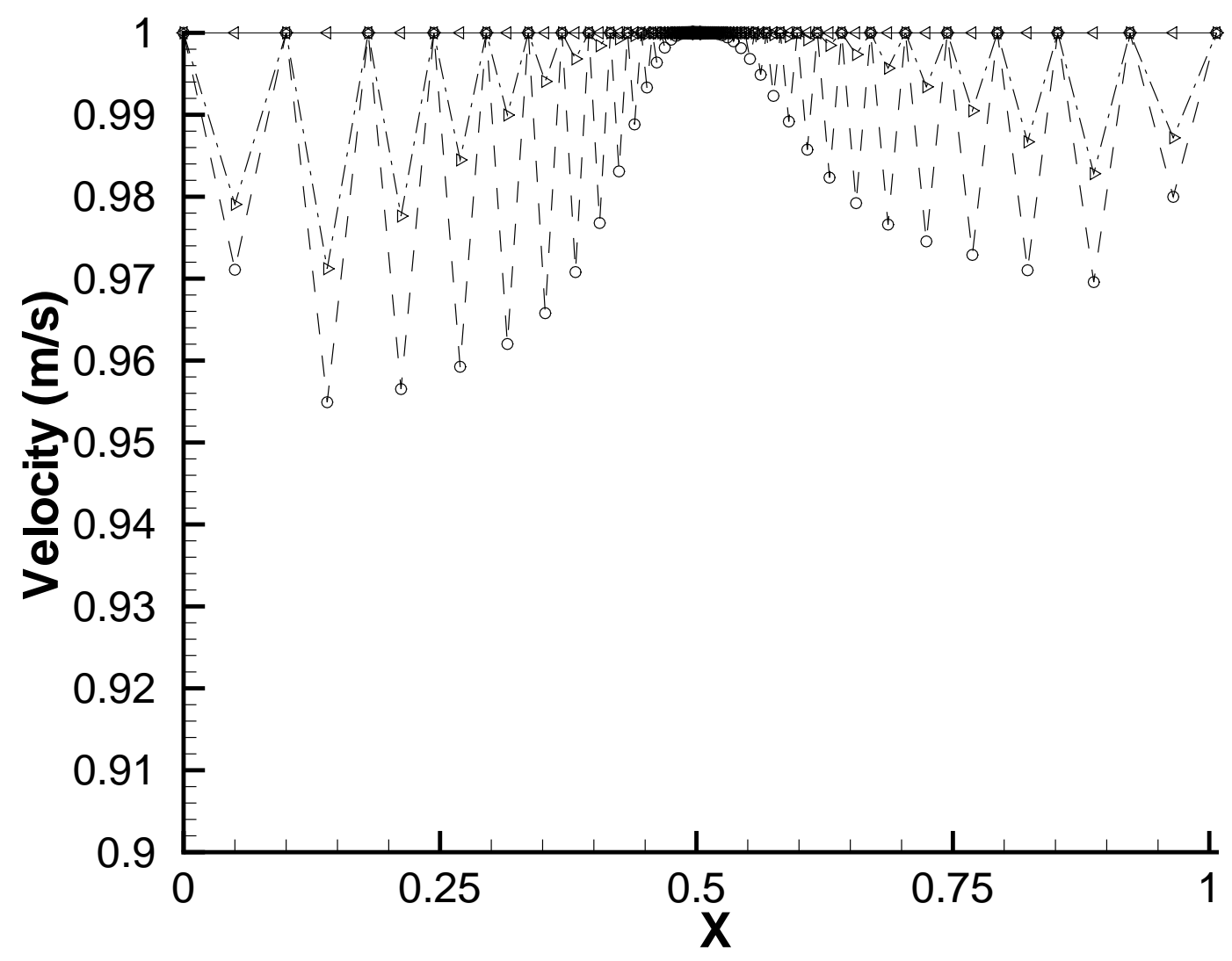

Fig. A.9. Velocity for the second case. $\bigcirc$ Choi inconsistent $\Delta t=10^{-4} ; \triangleright$ Choi inconsistent $\Delta t=10^{-3} ; \triangleleft$ Choi inconsistent $\Delta t=10^{+5}$; 


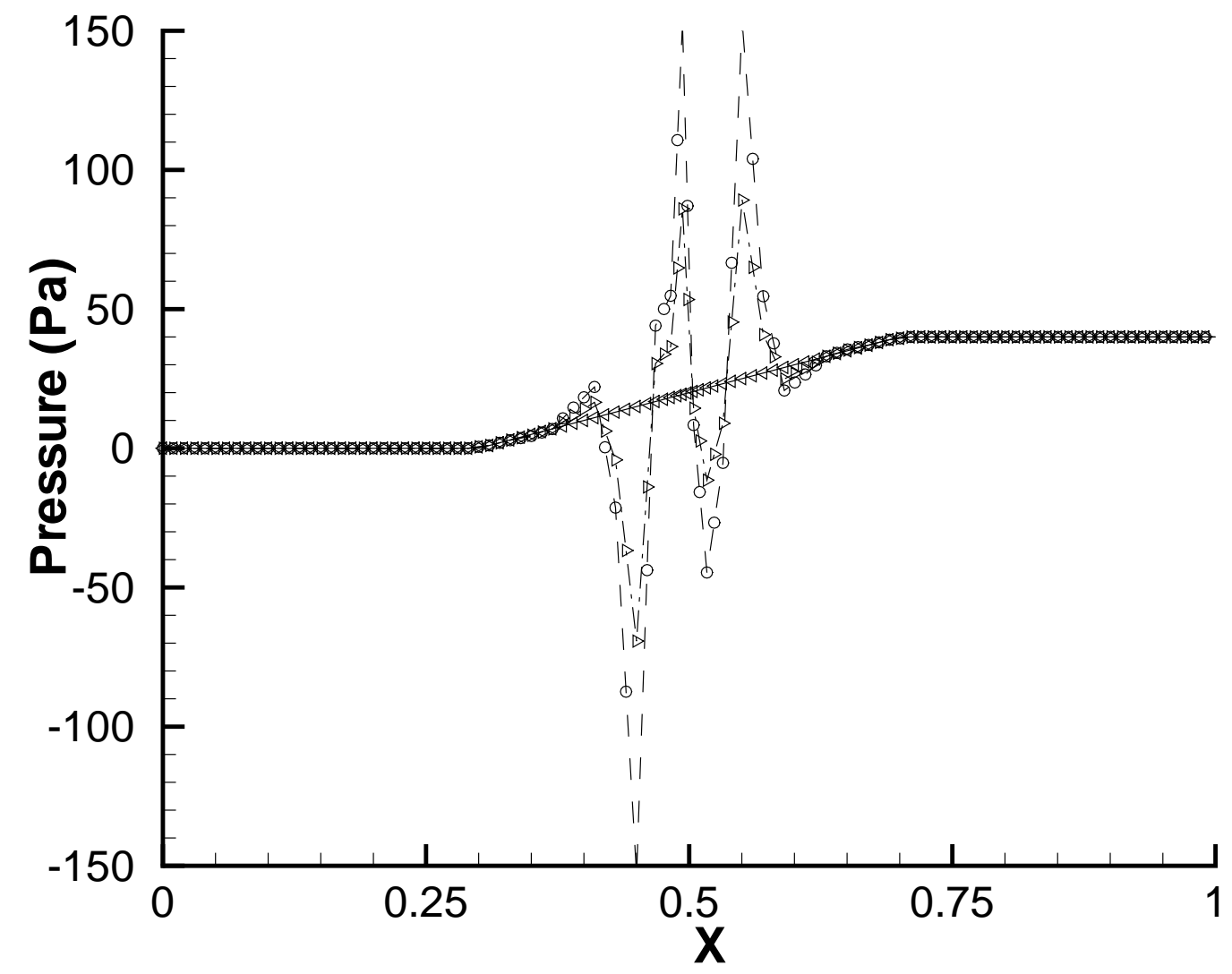

Fig. A.10. Pressure for the third case. $\bigcirc$ Kadja mass-consistent $\Delta t=5 \cdot 10^{-7}$; $\triangle$ Kadja mass-consistent $\Delta t=10^{-6} ; \triangleleft$ Kadja $\Delta V_{e}=\overline{\Delta V_{i}^{e}}, \Delta t=10^{-6}$; 


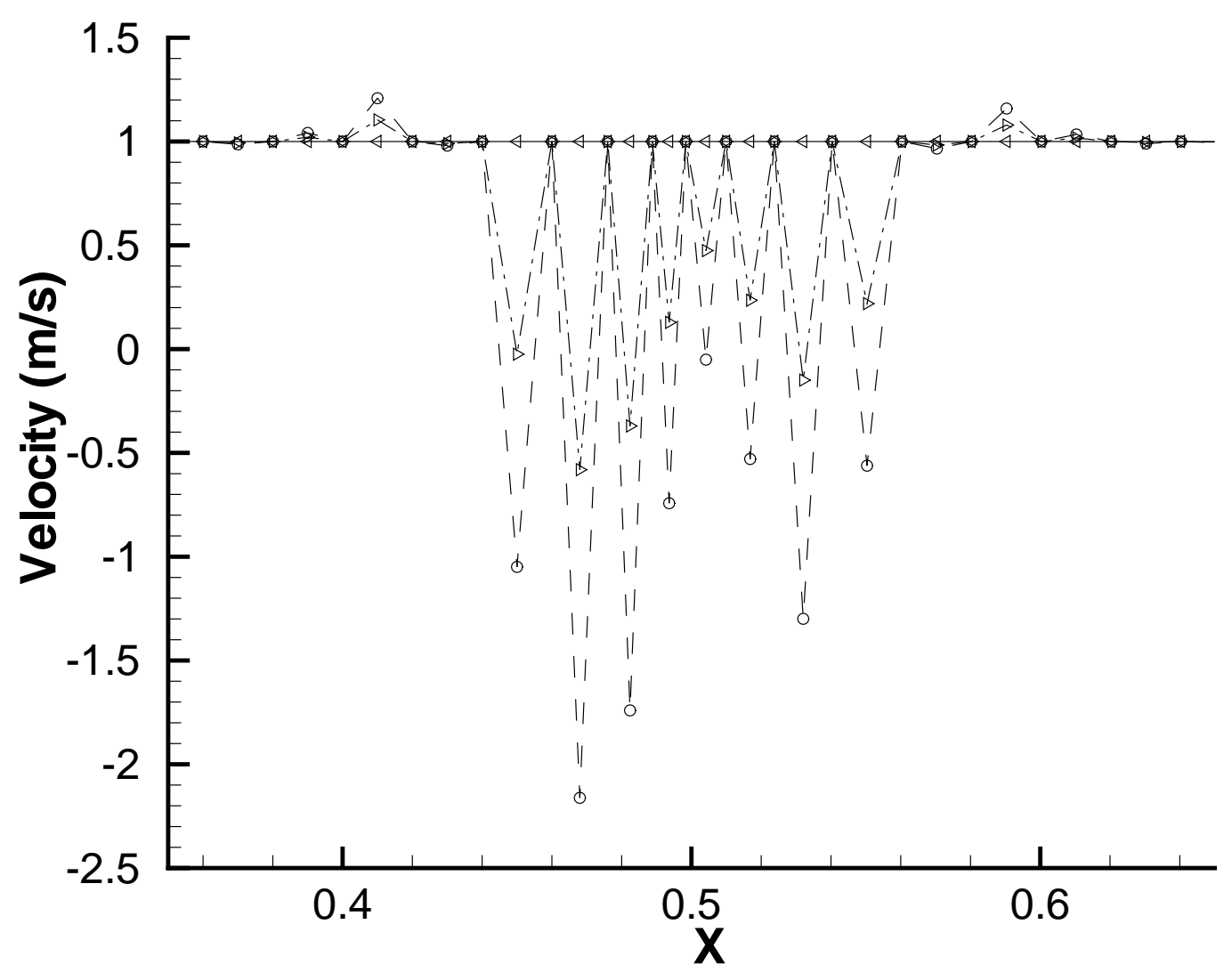

Fig. A.11. Velocity for the third case. $\bigcirc$ Kadja mass-consistent $\Delta t=5 \cdot 10^{-7}$; $\triangle$ Kadja mass-consistent $\Delta t=10^{-6} ; \triangleleft$ Kadja $\Delta V_{e}=\overline{\Delta V_{i}^{e}}, \Delta t=10^{-6}$; 